\title{
A General Methodology for the Enantioselective Synthesis of 1-Substituted Tetrahydroisoquinoline Alkaloids
}

\author{
Mercedes Amat, ${ }^{[\mathrm{a}]}$ Viviane Elias, ${ }^{[\mathrm{a}]}$ Núria Llor, ${ }^{[\mathrm{a}]}$ Fabiana Subrizi, ${ }^{[\mathrm{a}]}$ Elies Molins, ${ }^{[\mathrm{b}]}$ \\ and Joan Bosch ${ }^{[a]}$
}

Keywords: Alkaloids / Tetrahydroisoquinolines / Lactams / Phenylglycinol / $\alpha$-Amidoalkylation

Starting from tricyclic lactam 2 , which is easily accessible by cyclocondensation of $\delta$-oxoester 1 with $(R)$-phenylglycinol, a threestep synthetic route to enantiopure 1-substituted tetrahydroisoquinolines, including 1-alkyl-, 1-aryl-, and 1-benzyl- tetrahydroisoquinoline alkaloids as well as the tricyclic alkaloid (-)-crispine A, has been developed. The key step is a stereoselective $\alpha$-amidoalkylation reaction using the appropriate Grignard reagent. [a] Laboratory of Organic Chemistry, Faculty of Pharmacy, and Institute of Biomedicine (IBUB), University of Barcelona, Av. Joan XXIII s/n, 08028 Barcelona (Spain)

Fax: (+34) 934-024-539

E-mail:amat@ub.edu

[b] Institut de Ciència de Materials de Barcelona (CSIC)

\section{Introduction}

The tetrahydroisoquinoline ring system is present in numerous structurally diverse natural products exhibiting a wide range of biological and pharmacological activities. ${ }^{[1]}$ In particular, simple 1substituted tetrahydroisoquinolines are of great interest not only as alkaloids themselves but also as useful key intermediates in the synthesis of more complex alkaloids. This has stimulated the development of a number of methodologies aimed at the enantioselective synthesis of 1 -substituted tetrahydroisoquinoline derivatives $^{[2]}$ (Figure 1).

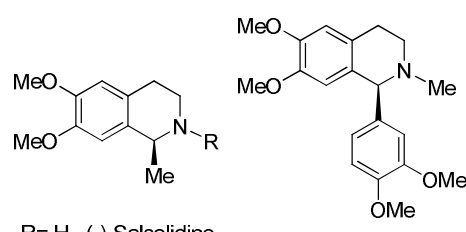

$\mathrm{R}=\mathrm{H} \quad(-)$-Salsolidine $\mathrm{R}=\mathrm{Me}(-)$-Carnegine

(+)-Cryptostyline II

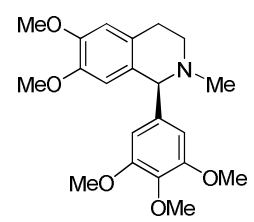

(+)-Cryptostyline III

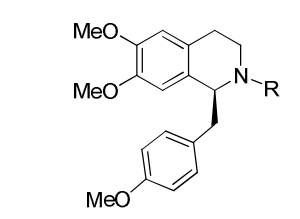

$\mathrm{R}=\mathrm{H}(-)-\mathrm{O}, \mathrm{O}-$ Dimethylcoclaurine $\mathrm{R}=\mathrm{Me}(+)-\mathrm{O}-$ Methylarmepavine

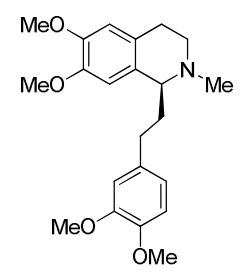

(+)-Homolaudanosine
Figure 1. Selected 1-substituted tetrahydroisoquinoline alkaloids.
In previous work we have demonstrated that phenylglycinolderived oxazolopiperidone lactams are versatile scaffolds that allow the regio- and stereocontrolled introduction of substituents at the different positions of the piperidine ring, thus providing access to enantiopure substituted piperidines bearing virtually any type of substitution pattern, as well as to quinolizidine, indolizidine, decahydroquinoline, and complex piperidine-containing indole alkaloids $^{[3]}$ (Scheme 1)

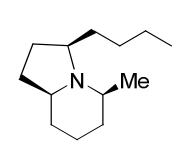

$$
\text { (+)-Monomorine }
$$<smiles>Fc1ccc([C@@H]2CCNCC2COc2ccc3c(c2)OCO3)cc1</smiles><smiles>CCCC1CCCC(C)N1</smiles>

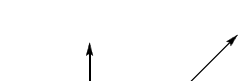
(2R,6S)-Dihydropinidine

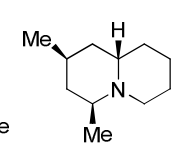

(-)-Cermizine C 
from an appropriate benzo-fused oxazolopiperidone lactam and the subsequent stereocontrolled introduction of the substituent at the 1position of the tetrahydroisoquinoline ring by an asymmetric $\alpha$ amidoalkylation reaction. ${ }^{[5]}$

Tricyclic lactam 2 was envisaged as the pivotal intermediate of our synthesis. It was prepared in $52 \%$ yield by cyclocondensation of aldehyde ester $\mathbf{1}^{[6]}$ with $(R)$-phenylglycinol in refluxing toluene in the presence of a catalytic amount of $p-\mathrm{TsOH}$ (Scheme 2). The absolute configuration of lactam $\mathbf{2}$ was unambiguously determined by X-ray crystallographic analysis. ${ }^{[7]}$ Minor amounts $(6 \%)$ of the lactam epi-2, epimeric at the 2-position of the oxazolidine ring, were also formed.

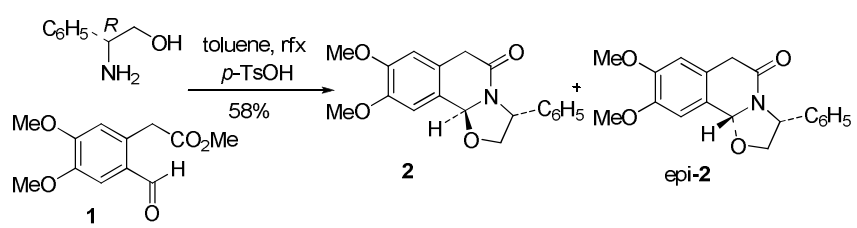

Scheme 2. Preparation of the key tricyclic lactam 2.

In contrast with related cis-oxazolopiperidone lactams, ${ }^{[8]}$ the minor cis lactam epi-2 did not undergo epimerization under acidic conditions (1.2 N HCl, $\mathrm{MeOH}$, r.t), isoquinolone 3 and trace amounts of dimer $\mathbf{4}$ being formed instead. This dimer was formed in $49 \%$ yield after a prolonged acidic treatment $(1.2 \mathrm{~N} \mathrm{HCl}, \mathrm{MeOH}$, reflux, $66 \mathrm{~h}$ ) of isoquinolone 3 .

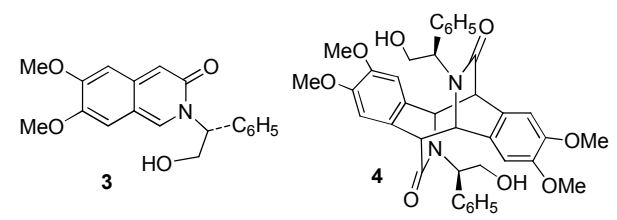

Initial attempts to carry out the $\alpha$-amidoalkylation reaction with a higher order cyanocuprate $\left[\mathrm{Me}_{2} \mathrm{Cu}(\mathrm{CN}) \mathrm{Li}_{2}\right]$ in the presence of $\mathrm{BF}_{3} . \mathrm{Et}_{2} \mathrm{O}^{[9]}$ resulted in failure, leading exclusively to isoquinolone 3. However, treatment of lactam 2 with an excess (3 equiv.) of methylmagnesium chloride at $5{ }^{\circ} \mathrm{C}$ stereoselectively led to the expected 1-substituted tetrahydroisoquinolone 5a in $61 \%$ yield
(Table 1). ${ }^{[10]}$ Isoquinolone 3 was formed as a by-product $(17 \%)$. Higher temperatures resulted in the formation of increasing amounts of $\mathbf{3}$, whereas when the reaction was carried out at a lower temperature the starting lactam was recovered to a considerable extent.

The observed retention of the configuration of the reactive methine carbon can be rationalized by considering that the Grignard reagent coordinates with the oxygen atom of the oxazolidine ring and that the subsequent intramolecular delivery of the alkyl group occurs on the same face of the $\mathrm{C}-\mathrm{O}$ bond of the incipient acyl iminium salt (Figure 2). ${ }^{[11]}$

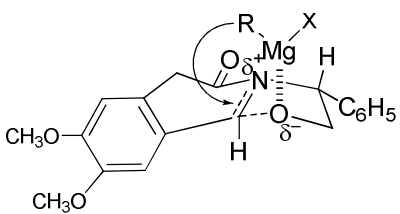

Figure 2. Stereochemical outcome of the $\alpha$-amidoalkylation reaction.

As in related cis-substituted oxazolopiperidones, ${ }^{[1]]}$ the minor $c i s$ lactam epi-2 was more reluctant to undergo $\alpha$-amidoalkylation ${ }^{[12]}$ than the trans isomer 2 and, on treatment with $\mathrm{MeMgCl}$, led to isoquinolone $\mathbf{3}$ as the major product $(50 \%)$, 2methyltetrahydroisoquinoline $\mathbf{5 a}$ being formed in low yield (35\%).

Removal of the phenylethanol moiety from lactam 5a was accomplished in excellent yield with sodium in liquid ammonia to give the $N$-unsubstituted lactam 6a. A subsequent reduction with borane generated in situ from $\mathrm{NaBH}_{4}$ and iodine completed the enantioselective synthesis of $(-)$-salsolidine $\mathbf{7 a} .^{[13]}$ Taking into account previous correlations, this synthesis also constitutes a formal synthesis of the alkaloid (-)-carnegine. ${ }^{[14]}$

The above protocol provides general access to 1-alkyl substituted tetrahydroisoquinolines. Thus, reaction of lactam $\mathbf{2}$ with ethylmagnesium bromide stereoselectively afforded ( $82 \%$ yield) lactam $\mathbf{5 b}$, which was then debenzylated and converted to $(S)-1$ ethyl-1,2,3,4-tetrahydroisoquinoline $\mathbf{7 b}$ in good overall yield, as in the above methyl series.

Table 1. Enantioselective synthesis of 1-substituted tetrahydroisoquinolines.

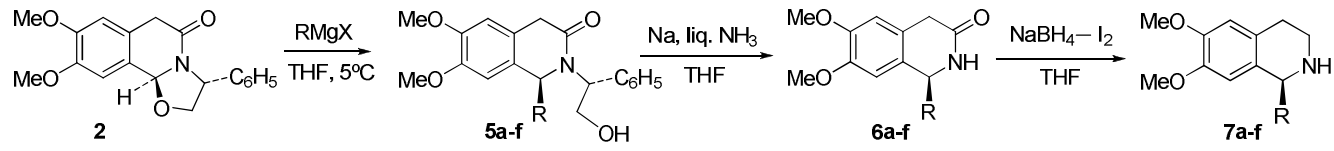

\begin{tabular}{cccccc}
\hline & $\mathbf{R}$ & $\mathbf{X}$ & Yield of 5 [\%] & Yield of 6 [\%] & Yield of 7 [\%] \\
\hline $\mathbf{a}$ & $\mathrm{Me}$ & $\mathrm{Cl}$ & 61 & 85 & 70 \\
$\mathbf{b}$ & $\mathrm{Et}$ & $\mathrm{Br}$ & 82 & 92 & 60 \\
$\mathbf{c}$ & $\mathrm{C}_{6} \mathrm{H}_{5}$ & $\mathrm{Cl}$ & 67 & - & - \\
$\mathbf{d}$ & $3,4-(\mathrm{MeO})_{2} \mathrm{C}_{6} \mathrm{H}_{3}$ & $\mathrm{Br}$ & 54 & 77 & 69 \\
$\mathbf{e}$ & $3,4,5-(\mathrm{MeO})_{3} \mathrm{C}_{6} \mathrm{H}_{2}$ & $\mathrm{Br}$ & 49 & 87 & 58 \\
$\mathbf{f}$ & $(p-\mathrm{MeO}) \mathrm{C}_{6} \mathrm{H}_{4} \mathrm{CH}_{2}$ & $\mathrm{Cl}$ & 63 & 85 & 59 \\
$\mathbf{g}$ & $\mathrm{C}_{6} \mathrm{H}_{5} \mathrm{CH}_{2} \mathrm{CH}_{2}$ & $\mathrm{Br}$ & 67 & 79 & 77 \\
$\mathbf{h}$ & $\mathrm{CH}_{2}=\mathrm{CHCH}_{2}$ & $\mathrm{Br}$ & 42 & 90 & - \\
$\mathbf{i}$ & $-\mathrm{Br}$ & 45 & 92 & -
\end{tabular}


With the aim of demonstrating the potential of the methodology for the synthesis of 1-aryl-, 1-benzyl-, and 1-phenethyl tetrahydroisoquinoline alkaloids, we applied the above three-step sequence from lactam 2 using a variety of aryl-, benzyl-, and phenethylmagnesium halides. The results are summarized in Table 1 (entries $\mathbf{c - g}$ ). In all cases the $\alpha$-amidoalkylation reaction took place stereoselectively to give a single 1-substituted tetrahydroisoquinolone derivative $(\mathbf{5} \mathbf{c}-\mathbf{g}) \cdot{ }^{[15]}$

Although the reductive cleavage of the exocyclic benzylic $\mathrm{C}-\mathrm{N}$ bond of the 2-phenyl derivative $\mathbf{5 c}$ with $\mathrm{Na}$ /liq. $\mathrm{NH}_{3}$ occurred with concomitant cleavage of the doubly benzylic endocyclic $\mathrm{C}-\mathrm{N}$ bond to give 2-benzyl-4,5-dimethoxyphenylacetamide (8), a similar reduction from the methoxyphenyl substituted tetrahydroisoquinolones $\mathbf{5 d}$ and $\mathbf{5 e}$ satisfactorily led to the respective $N$-unsusbtituted lactams $\mathbf{6 d}$ and $\mathbf{6 e}$ in excellent yield. A subsequent reduction of the lactam carbonyl of $\mathbf{6 d}$ led to (-)norcryptostyline II (7d), which constitutes a formal synthesis of the alkaloid (+)-cryptostyline II. ${ }^{[16]}$ Similarly, lactam 6e was converted to (-)-norcryptostyline III (7e), a known precursor of the alkaloid $(+)$-cryptostyline III. ${ }^{[17]}$

The same set of sequential reductions $\left(\mathrm{Na} /\right.$ liq. $\mathrm{NH}_{3}$ and then $\mathrm{NaBH}_{4}-\mathrm{I}_{2}$ ) was used to convert 2-benzyl derivative $\mathbf{5 f}$ to $(-)-\mathrm{O}, \mathrm{O}-$ dimethylcoclaurine (7f). ${ }^{[18]}$ Taking into account previous transformations, this synthesis also constitutes a formal synthesis of the alkaloids (+)-O-methylarmepavine,$^{[18 a, b]}$ zanoxyline $^{[19]}$ and (-)-demethylcoclaurine [(-)-higenamine $].^{[18 \mathrm{e}]}$

Similarly, the usefulness of this methodology in the synthesis of 1-phenethyltetrahydroisoquinolines was demonstrated by the preparation of $\mathbf{7} \mathbf{g}^{[20]}$ from the $\alpha$-amidoalkylation product $\mathbf{5 g}$.

The procedure allows the preparation of tetrahydroisoquinolines and tetrahydroisoquinolones bearing a functionalized $\mathrm{C}-1$ substituent, for instance allyl ${ }^{[21]}$ or 2-(1,3-dioxan-2-yl)ethyl (Table 1 , entries $\mathbf{h}, \mathbf{i}),{ }^{[22]}$ which can open access to more complex tetrahydroisoquinoline alkaloids embodying an additional ring. This was exemplified with the synthesis of the pyrrolo[2,1$g$ ]isoquinoline alkaloid crispine A. The three-carbon fragment required to assemble the pyrrolidine ring was incorporated in the $\alpha$-amidoalkylation step by reaction of lactam 2 with the Grignard reagent derived from 2-(2-bromoethyl)-1,3-dioxane (Scheme 3) to give $5 \mathbf{i}$. In this synthesis, the lactam carbonyl was reduced prior to debenzylation to give tetrahydroisoquinoline 9 in excellent yield. A subsequent catalytic hydrogenation under acidic conditions brought about the hydrogenolysis of the exocyclic benzylic $\mathrm{C}-\mathrm{N}$ bond, deprotection of the acetal function, and closure of the pyrrolidine ring by reductive amination, directly leading to crispine $\mathrm{A}^{[23]}$ in $74 \%$ yield.

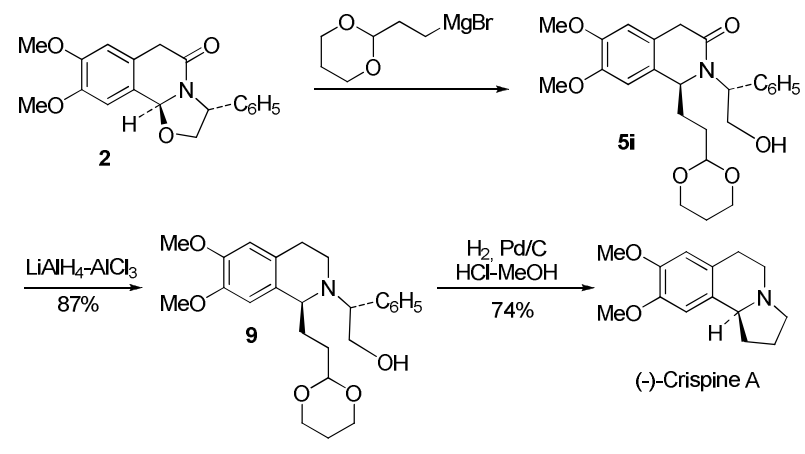

Scheme 3. Enantioselective synthesis of (-)-crispine A.

\section{Conclusions}

Tricyclic $(R)$-phenylglycinol-derived lactam $\mathbf{2}$ has proven to be a useful scaffold that provides general access to enantiopure 1substituted tetrahydroisoquinoline derivatives, including 1-alkyl-, 1-aryl-, and 1-benzyltetrahydroisoquinoline alkaloids as well as more complex alkaloids bearing the tetrahydroisoquinoline moiety (Scheme 4). The enantioselective synthesis of 1benzyltetrahydroisoquinolines is of particular interest because these derivatives not only play a pivotal role in the biosynthesis of numerous alkaloids with a variety of skeletal types (e.g. aporphines, cularines, protoberberines, and pavines) but have also been used as key synthetic precursors of such alkaloids. ${ }^{[24]}$

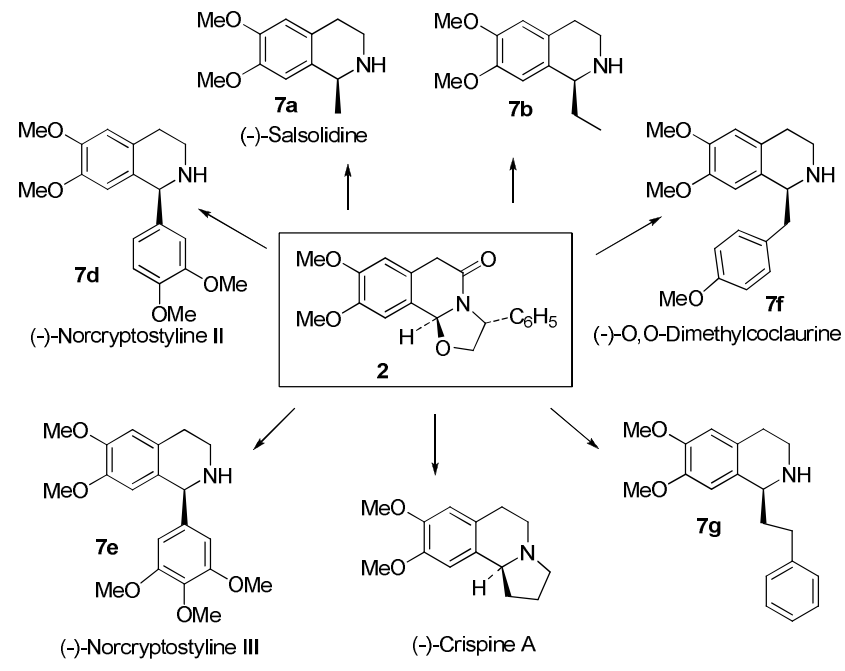

Scheme 4. Enantiopure 1-substituted tetrahydroisoquinolines prepared from the common scaffold 2.

\section{Experimental Section}

$(3 R, 10 \mathrm{~b} S)-8,9-D i m e t h o x y-5-0 x 0-3-p h e n y l-2,3,6,10 \mathrm{~b}-$ tetrahydro$5 H$-oxazolo[2,3-a]isoquinoline (2): To a mixture of aldehyde-ester $\mathbf{1}^{[6]}(993 \mathrm{mg}, 4.2 \mathrm{mmol})$ and $(R)$-phenylglycinol $(690 \mathrm{mg}, 5.0$ mmol) in anhydrous toluene $(45 \mathrm{~mL})$ containing $4 \AA$ molecular sieves was added a catalytic amount of $p$-TsOH. The mixture was heated at reflux for $18 \mathrm{~h}$ with azeotropic elimination of water produced by a Dean-Stark apparatus. The resulting suspension was concentrated under reduced pressure to give a yellow foam. Flash 
chromatography $\left(\mathrm{Et}_{2} \mathrm{O}\right.$ to EtOAc) afforded lactam $2(710 \mathrm{mg}, 52 \%)$ and the 10b-epimer epi-2 (82 mg, 6\%). 2: White solid; m.p. 135$137^{\circ} \mathrm{C}\left(\mathrm{Et}_{2} \mathrm{O}\right) .[\alpha]^{22}{ }_{\mathrm{D}}=-136.6\left(c=1.0, \mathrm{CHCl}_{3}\right)$. IR $(\mathrm{KBr})$ : $v=1665 \mathrm{~cm}^{-1} .{ }^{1} \mathrm{H}$ NMR $\left(400 \mathrm{MHz}, \mathrm{CDCl}_{3}, 25^{\circ} \mathrm{C}\right): \delta=3.52(\mathrm{~d}, J=$ $19.2 \mathrm{~Hz}, 1 \mathrm{H}, \mathrm{H}-6$ ), 3.73 (dd, $J=19.2,2.2 \mathrm{~Hz}, 1 \mathrm{H}, \mathrm{H}-6$ ), 3.88 (s, $\left.3 \mathrm{H}, \mathrm{OCH}_{3}\right), 3.91\left(\mathrm{~s}, 3 \mathrm{H}, \mathrm{OCH}_{3}\right), 4.02(\mathrm{dd}, J=9.0,7.5 \mathrm{~Hz}, 1 \mathrm{H}, \mathrm{H}-$ 2), 4.58 (dd, $J=9.0,7.5 \mathrm{~Hz}, 1 \mathrm{H}, \mathrm{H}-2), 5.38$ (t, $J=7.5,1 \mathrm{H}, \mathrm{H}-3)$, 6.02 (d, $J=2.2 \mathrm{~Hz}, 1 \mathrm{H}, \mathrm{H}-10 \mathrm{~b}), 6.66$ (s, 1H, H-10), 6.99 (s, 1H, H7), 7.35 (m, 5H, ArH) ppm. ${ }^{13} \mathrm{C} \mathrm{NMR}\left(100.6 \mathrm{MHz}, \mathrm{CDCl}_{3}, 25^{\circ} \mathrm{C}\right)$ : $\delta=36.9(\mathrm{C}-6), 56.0\left(\mathrm{OCH}_{3}\right), 56.1\left(\mathrm{OCH}_{3}\right), 58.6(\mathrm{C}-3), 72.7(\mathrm{C}-2)$, 87.8 (C-10b), 107.7 (C-10), 109.8 (C-7), 122.5 (C-6a), 123.1 (C10a), 126.0 (C-o), 127.7 (C-p), 128.8 (C-m), 139.3 (C-i), 148.4 (C8), 149.7 (C-9), 166.2 (CO) ppm. HRMS calcd. for $\mathrm{C}_{19} \mathrm{H}_{19} \mathrm{NO}_{4}[\mathrm{M}$ $+\mathrm{H}]^{+}$: 326.1386; found 326.1382. $\mathrm{C}_{19} \mathrm{H}_{19} \mathrm{NO}_{4}$ (325.36): calcd. C 70.14, H 5.89, N 4.31; found C 70.27, H 5.87, N 4.08. epi-2: $[\alpha]^{22}$ $=+62.5\left(c=1.0, \mathrm{CHCl}_{3}\right)$. IR $(\mathrm{KBr}): \mathrm{v}=1667 \mathrm{~cm}^{-1} .{ }^{1} \mathrm{H}$ NMR $(300$ $\left.\mathrm{MHz}, \mathrm{CDCl}_{3}, 25^{\circ} \mathrm{C}\right): \delta=3.45(\mathrm{~d}, J=18.9 \mathrm{~Hz}, 1 \mathrm{H}, \mathrm{H}-6), 3.60$ (dd, $J$ $=18.9,2.1 \mathrm{~Hz}, 1 \mathrm{H}, \mathrm{H}-6), 3.90\left(\mathrm{~s}, 3 \mathrm{H}, \mathrm{OCH}_{3}\right), 3.94\left(\mathrm{~s}, 3 \mathrm{H}, \mathrm{OCH}_{3}\right)$, 4.20 (dd, $J=9.0,0.9 \mathrm{~Hz}, 1 \mathrm{H}, \mathrm{H}-2), 4.47$ (dd, $J=9.0,6.3 \mathrm{~Hz}, 1 \mathrm{H}$, $\mathrm{H}-2), 5.10(\mathrm{~d}, J=6.3 \mathrm{~Hz}, 1 \mathrm{H}, \mathrm{H}-3), 5.90(\mathrm{~d}, J=2.1 \mathrm{~Hz}, 1 \mathrm{H}, \mathrm{H}-$ 10b), 6.71 (s, 1H, H-10), 7.10 (s, 1H, H-7), 7.15-7.25 (m, 5H, ArH) ppm. ${ }^{13} \mathrm{C}$ NMR $\left(75.6 \mathrm{MHz}, \mathrm{CDCl}_{3}, 25^{\circ} \mathrm{C}\right): \delta=38.1(\mathrm{C}-6), 55.9$ $\left(\mathrm{OCH}_{3}\right), 56.0\left(\mathrm{OCH}_{3}\right), 58.6(\mathrm{C}-3), 74.8(\mathrm{C}-2), 87.3(\mathrm{C}-10 \mathrm{~b}), 106.5$ (C-10), 110.1 (C-7), 123.0 (C-6a), 125.0 (C-10a), 125.9 (C-o), 127.4 (C-p), 128.4 (C-m), 140.5 (C-i), 148.0 (C-8), 149.2 (C-9), 165.2 (CO) ppm. HRMS calcd. for $\mathrm{C}_{19} \mathrm{H}_{19} \mathrm{NO}_{4}[\mathrm{M}+\mathrm{H}]^{+}$: 326.1386 ; found 326.1382 .

Dimer 4: A solution of $1.2 \mathrm{M} \mathrm{HCl}$ in $\mathrm{MeOH}(6 \mathrm{~mL})$ was added to a solution of isoquinolone $3(96 \mathrm{mg}, 0.3 \mathrm{mmol})$ in $\mathrm{MeOH}(1 \mathrm{~mL})$. The mixture was heated at reflux for $66 \mathrm{~h}$. The solvent was removed, and the resulting solid was diluted with EtOAc. The solution was washed with saturated aqueous $\mathrm{Na}_{2} \mathrm{CO}_{3}$. The organic phase was dried and concentrated to give a residue, which was chromatographed (7:3 $\mathrm{Et}_{2} \mathrm{O}-\mathrm{EtOAc}$ to EtOAc) to afford 4 (47 mg, $49 \%)$ as a yellow oil. ${ }^{1} \mathrm{H}$ NMR $\left(400 \mathrm{MHz}, \mathrm{CDCl}_{3}, 25^{\circ} \mathrm{C}\right): \delta=3.48$ $\left(\mathrm{s}, 6 \mathrm{H}, 2 \mathrm{OCH}_{3}\right), 3.64\left(\mathrm{~s}, 6 \mathrm{H}, 2 \mathrm{OCH}_{3}\right), 4.27$ (dd, $J=13.0,2.8 \mathrm{~Hz}$, $\left.2 \mathrm{H}, 2 \mathrm{CH}_{2} \mathrm{OH}\right), 4.43(\mathrm{~d}, J=10.8 \mathrm{~Hz}, 2 \mathrm{H}, 2 \mathrm{CHCO}), 4.44$ (dd, $J=$ 13.0, $\left.3.6 \mathrm{~Hz}, 2 \mathrm{H}, 2 \mathrm{CH}_{2} \mathrm{OH}\right), 4.79$ (d, $\left.J=11.2 \mathrm{~Hz}, 2 \mathrm{H}, 2 \mathrm{CHNCO}\right)$, $5.68\left(\mathrm{~s}, 2 \mathrm{H}, 2 \mathrm{CH}_{3} \mathrm{OCCH}\right), 5.73(\mathrm{t}, J=2.9 \mathrm{~Hz}, 2 \mathrm{H}, \mathrm{CHAr}), 6.26(\mathrm{~s}$, $\left.2 \mathrm{H}, 2 \mathrm{CH}_{3} \mathrm{OCCH}\right), 7.08(\mathrm{~d}, J=6.4 \mathrm{~Hz}, 4 \mathrm{H}, \mathrm{ArH}), 7.14-7.23(\mathrm{~m}, 6 \mathrm{H}$, $\mathrm{ArH}) \mathrm{ppm} .{ }^{13} \mathrm{C} \mathrm{NMR}\left(100.6 \mathrm{MHz}, \mathrm{CDCl}_{3}, 25^{\circ} \mathrm{C}\right): \delta=55.9\left(\mathrm{OCH}_{3}\right)$, $56.0\left(\mathrm{OCH}_{3}\right), 56.9(\mathrm{CHCO}), 58.5$ ( $\left.\mathrm{CHNCO}\right), 58.7$ (CHAr), 62.1 $\left(\mathrm{CH}_{2} \mathrm{OH}\right), 108.6\left(\mathrm{CH}_{3} \mathrm{OCCH}\right), 110.3\left(\mathrm{CH}_{3} \mathrm{OCCH}\right), 127.7(\mathrm{C}-p)$, 128.4 (C-o), 128.7 (CCHCO), 128.9 (C-m), 131.5 (CCHN), 136.4 (C-i), $147.5\left(\mathrm{CH}_{3} \mathrm{OC}\right), 147.9\left(\mathrm{CH}_{3} \mathrm{OC}\right), 176.0(\mathrm{CO})$ ppm. HRMS calcd. for $\mathrm{C}_{38} \mathrm{H}_{38} \mathrm{~N}_{2} \mathrm{O}_{8}[\mathrm{M}+\mathrm{H}]^{+}$: 651.2706; found 651.2697.

General Procedure for the $\boldsymbol{\alpha}$-Amidoalkylation Reaction: The Grignard reagent (3.0 equiv) was added to a cooled $\left(5^{\circ} \mathrm{C}\right)$ solution of oxazolopiperidone 2 ( 1 equiv) in THF, and the mixture was stirred at this temperature until the disappearance of the starting material was observed by TLC. The reaction was quenched by the addition of water, and the mixture extracted with EtOAc. The combined extracts were dried and concentrated to give the 1substituted tetrahydroisoquinolones after flash chromatography.

(1S)-2-[(1R)-2-Hydroxy-1-phenylethyl]-6,7-dimethoxy-1methyl-3-oxo-1,2,3,4-tetrahydroisoquinoline (5a): Following the above general procedure (reaction time $1.5 \mathrm{~h}$ ), from lactam 2 (100 $\mathrm{mg}, 0.31 \mathrm{mmol}$ ) and methylmagnesium chloride (3M in THF, 0.31 $\mathrm{mL}, 0.92 \mathrm{mmol})$ in THF $(12.5 \mathrm{~mL})$ a brown oil was obtained. Flash chromatography (7:3 $\mathrm{Et}_{2} \mathrm{O}-\mathrm{EtOAc}$, increasing polarity and 9:1 EtOAc-EtOH) gave 5a (64 mg, 61\%) as a yellow oil and isoquinolone $3(17 \mathrm{mg}, 17 \%)$ as a yelow-green foam. 5a: $[\alpha]^{22}{ }_{\mathrm{D}}=$ $+23.5\left(c=1.0, \mathrm{CHCl}_{3}\right)$. IR $(\mathrm{KBr}): v=1629,3400 \mathrm{~cm}^{-1} .{ }^{1} \mathrm{H}$ NMR $\left(400 \mathrm{MHz}, \mathrm{CDCl}_{3}, 25^{\circ} \mathrm{C}\right): \delta=1.39\left(\mathrm{~d}, J=7.2 \mathrm{~Hz}, 3 \mathrm{H}, \mathrm{CH}_{3}\right), 3.60$ (d, $J=18.9 \mathrm{~Hz}, 1 \mathrm{H}, \mathrm{H}-4), 3.76$ (d, $J=18.9 \mathrm{~Hz}, 1 \mathrm{H}, \mathrm{H}-4), 3.77$ (s, $\left.3 \mathrm{H}, \mathrm{OCH}_{3}\right), 3.86\left(\mathrm{~s}, 3 \mathrm{H}, \mathrm{OCH}_{3}\right), 4.28(\mathrm{q}, J=7.2 \mathrm{~Hz}, 1 \mathrm{H}, \mathrm{H}-1)$, $4.29\left(\mathrm{dd}, J=12.0,8.4 \mathrm{~Hz}, 1 \mathrm{H}, \mathrm{CH}_{2} \mathrm{OH}\right), 4.30(\mathrm{dd}, J=12.0,5.2 \mathrm{~Hz}$, $\left.1 \mathrm{H}, \mathrm{CH}_{2} \mathrm{OH}\right), 5.70$ (dd, $\left.J=8.4,5.2 \mathrm{~Hz}, 1 \mathrm{H}, \mathrm{CHAr}\right), 6.38(\mathrm{~s}, 1 \mathrm{H}, \mathrm{H}-$ 8), $6.66(\mathrm{~s}, 1 \mathrm{H}, \mathrm{H}-5), 7.18-7.30(\mathrm{~m}, 5 \mathrm{H}, \mathrm{ArH}) \mathrm{ppm} .{ }^{13} \mathrm{C} \mathrm{NMR}$ $\left(100.6 \mathrm{MHz}, \mathrm{CDCl}_{3}, 25^{\circ} \mathrm{C}\right): \delta=23.2\left(\mathrm{CH}_{3}\right), 37.3(\mathrm{C}-4), 55.6$ $\left(\mathrm{OCH}_{3}\right), 56.0\left(\mathrm{OCH}_{3}\right), 56.1(\mathrm{C}-1), 60.8(\mathrm{CHAr}), 63.3\left(\mathrm{CH}_{2} \mathrm{OH}\right)$, 107.7 (C-8), 110.3 (C-5), 123.1 (C-4a), 127.6 (C-o), 128.1 (C-p), 128.7 (C-m), 130.5 (C-8a), 136.8 (C-i), 148.3 (C-7), 147.8 (C-6), 171.7 (CO) ppm. HRMS calcd. for $\mathrm{C}_{20} \mathrm{H}_{23} \mathrm{NO}_{4}[\mathrm{M}+\mathrm{H}]^{+}$: 342.1699; found 342.1695. $\mathrm{C}_{20} \mathrm{H}_{23} \mathrm{NO}_{4} 1 / 4 \mathrm{CH}_{2} \mathrm{Cl}_{2}$ (362.64): calcd. C 67.07, H 6.53, N 3.86; found C 67.13, H 6.65, N 3.81. 3: IR $(\mathrm{KBr}): \mathrm{v}=1652,3269 \mathrm{~cm}^{-1} .{ }^{1} \mathrm{H}$ NMR $\left(400 \mathrm{MHz}, \mathrm{CDCl}_{3}, 25^{\circ} \mathrm{C}\right): \delta$ $=3.82\left(\mathrm{~s}, 3 \mathrm{H}, \mathrm{OCH}_{3}\right), 3.85\left(\mathrm{~s}, 3 \mathrm{H}, \mathrm{OCH}_{3}\right), 4.31(\mathrm{dd}, J=12.4,7.2$ $\left.\mathrm{Hz}, 1 \mathrm{H}, \mathrm{CH}_{2} \mathrm{OH}\right), 4.42$ (dd, $\left.J=12.4,4.4 \mathrm{~Hz}, 1 \mathrm{H}, \mathrm{CH}_{2} \mathrm{OH}\right), 6.12$ (s, $1 \mathrm{H}, \mathrm{H}-5), 6.26$ (s, 1H, H-8), 6.47 (dd, $J=7.2,4.4 \mathrm{~Hz}, 1 \mathrm{H}, \mathrm{CHAr}$ ), 6.59 (s, 1H, H-4), 7.33 (m, 5H, ArH), 7.95 (s, 1H, H-1) ppm. ${ }^{13} \mathrm{C}$ NMR $\left(100.6 \mathrm{MHz}, \mathrm{CDCl}_{3}, 25^{\circ} \mathrm{C}\right): \delta=55.7\left(\mathrm{OCH}_{3}\right), 55.8\left(\mathrm{OCH}_{3}\right)$, 60.7 (CHAr), 62.9 ( $\left.\mathrm{CH}_{2} \mathrm{OH}\right), 100.6$ (C-7), 103.6 (C-5), 107.6 (C-4), 114.2 (C-8a), 127.9 (C-m), 128.1 (C-p), 128.9 (C-o), 135.3 (C-1), 137.4 (C-i), 140.6 (C-6), 147.7 (C-7), 155.0 (C-4a), 160.5 (CO) ppm. EM (IQ $):$ m/z (\%): 326 (47); 325 (5); 206 (13); 138 (27); 122 (12) 121 (100).

(1S)-1-Ethyl-2-[(1R)-2-hydroxy-1-phenylethyl]-6,7-dimethoxy3-oxo-1,2,3,4-tetrahydroisoquinoline (5b): Following the general procedure (reaction time $6 \mathrm{~h}$ ), from lactam $2(400 \mathrm{mg}, 1.23 \mathrm{mmol})$ in THF $(10 \mathrm{~mL})$ and ethylmagnesium bromide $\left(3 \mathrm{M}\right.$ in $\mathrm{Et}_{2} \mathrm{O}, 1.23$ $\mathrm{mL}, 3.68 \mathrm{mmol})$ a residue was obtained. Flash chromatography $\left(1: 1 \mathrm{Et}_{2} \mathrm{O}-\mathrm{EtOAc}\right)$ gave $\mathbf{5 b}(354 \mathrm{mg}, 82 \%)$ as a yellow oil: $[\alpha]^{22}{ }_{\mathrm{D}}=$ $+34.4\left(c=1.0, \mathrm{CHCl}_{3}\right)$. IR $(\mathrm{KBr}): \mathrm{v}=1630,3388 \mathrm{~cm}^{-1} .{ }^{1} \mathrm{H} \mathrm{NMR}$ $\left(400 \mathrm{MHz}, \mathrm{CDCl}_{3}, 25^{\circ} \mathrm{C}\right): \delta=0.72\left(\mathrm{t}, J=7.2 \mathrm{~Hz}, 3 \mathrm{H}, \mathrm{CH}_{3}\right), 1.66-$ $1.77\left(\mathrm{~m}, 1 \mathrm{H}, \mathrm{CH}_{2}\right), 1.79-1.89\left(\mathrm{~m}, 1 \mathrm{H}, \mathrm{CH}_{2}\right), 3.57(\mathrm{~d}, J=18.0 \mathrm{~Hz}$, $1 \mathrm{H}, \mathrm{H}-4), 3.77$ (s, $\left.3 \mathrm{H}, \mathrm{OCH}_{3}\right), 3.79$ (d, $\left.J=18.0 \mathrm{~Hz}, 1 \mathrm{H}, \mathrm{H}-4\right), 3.86$ (s, $\left.3 \mathrm{H}, \mathrm{OCH}_{3}\right), 4.00$ (dd, $\left.J=9.6,3.2 \mathrm{~Hz}, 1 \mathrm{H}, \mathrm{H}-1\right), 4.23$ (dd, $J=$ 11.6, $\left.8.4 \mathrm{~Hz}, 1 \mathrm{H}, \mathrm{CH}_{2} \mathrm{OH}\right), 4.29(\mathrm{dd}, J=11.6,5.2 \mathrm{~Hz}, 1 \mathrm{H}$, $\mathrm{CH}_{2} \mathrm{OH}$ ), 5.66 (dd, $\left.J=8.4,5.2 \mathrm{~Hz}, 1 \mathrm{H}, \mathrm{CHAr}\right), 6.33$ (s, $\left.1 \mathrm{H}, \mathrm{H}-8\right)$, 6.67 (s, 1H, H-5), 7.10-7.30 (m, 5H, ArH) ppm. ${ }^{13} \mathrm{C}$ NMR (100.6 $\left.\mathrm{MHz}, \mathrm{CDCl}_{3}, 25^{\circ} \mathrm{C}\right): \delta=10.2\left(\mathrm{CH}_{3}\right), 29.1\left(\mathrm{CH}_{2}\right), 37.5(\mathrm{C}-4), 55.9$ $\left(\mathrm{OCH}_{3}\right), 56.1\left(\mathrm{OCH}_{3}\right), 61.3(\mathrm{C}-1), 61.5(\mathrm{CHAr}), 63.4\left(\mathrm{CH}_{2} \mathrm{OH}\right)$, 109.3 (C-8), 110.3 (C-5), 123.7 (C-4a), 127.7 (C-o), 127.8 (C-p), 127.8 (C-8a), 129.9 (C-m), 136.8 (C-i), 147.2 (C-7), 148.3 (C-6), 171.9 (CO) ppm. HRMS calcd. for $\mathrm{C}_{21} \mathrm{H}_{25} \mathrm{NO}_{4}[\mathrm{M}+\mathrm{H}]^{+}$: 356.1862; found 356.1872. $\mathrm{C}_{21} \mathrm{H}_{25} \mathrm{NO}_{4} .1 / 4 \mathrm{CHCl}_{3}$ (385.28): calcd. C 66.25, H 6.61, N 3.64; found C 66.13, H 6.64, N 3.53.

(1S)-2-[(1R)-2-Hydroxy-1-phenylethyl]-6,7-dimethoxy-3-oxo-1phenyl-1,2,3,4-tetrahydroisoquinoline (5c): Following the general procedure (reaction time $1 \mathrm{~h}$ ), from lactam 2 (500 mg, 1.54 $\mathrm{mmol})$ in THF $(10 \mathrm{~mL})$ and phenylmagnesium chloride $(2 \mathrm{M}$ in THF, $2.3 \mathrm{~mL}, 4.61 \mathrm{mmol}$ ) a residue was obtained. Flash chromatography $\left(\mathrm{Et}_{2} \mathrm{O}-\mathrm{EtOAc}\right.$ increasing polarity and 9:1 EtOAcEtOH) gave 5c (415 mg, 67\%) as a yellow oil and 3 (125 mg, 25\%). 
5c: $[\alpha]^{22}{ }_{D}=+16.7\left(c=1.0, \mathrm{CHCl}_{3}\right)$. IR $(\mathrm{KBr}): \mathrm{v}=1629,3388$ $\mathrm{cm}^{-1} .{ }^{1} \mathrm{H}$ NMR $\left(300 \mathrm{MHz}, \mathrm{CDCl}_{3}, 25^{\circ} \mathrm{C}\right): \delta=3.66(\mathrm{~d}, J=19.8 \mathrm{~Hz}$, $1 \mathrm{H}, \mathrm{H}-4), 3.71\left(\mathrm{~s}, 3 \mathrm{H}, \mathrm{OCH}_{3}\right), 3.80\left(\mathrm{~s}, 3 \mathrm{H}, \mathrm{OCH}_{3}\right), 3.84(\mathrm{~d}, J=$ $19.8 \mathrm{~Hz}, 1 \mathrm{H}, \mathrm{H}-4), 3.88-3.91$ (m, 2H, $\left.\mathrm{CH}_{2} \mathrm{OH}\right), 5.26$ (s, $\left.1 \mathrm{H}, \mathrm{H}-1\right)$, $5.96(\mathrm{t}, J=6.6 \mathrm{~Hz}, 1 \mathrm{H}, \mathrm{CHAr}), 6.41$ (s, 1H, H-8), 6.58 (s, 1H, H-5), 7.15-7.30 (m, 10H, ArH) ppm. ${ }^{13} \mathrm{C}$ NMR (75.4 $\mathrm{MHz}, \mathrm{CDCl}_{3}$, $\left.25^{\circ} \mathrm{C}\right): \delta=37.2(\mathrm{C}-4), 55.8\left(\mathrm{OCH}_{3}\right), 55.9\left(\mathrm{OCH}_{3}\right), 59.9(\mathrm{C}-1), 62.2$ $\left(\mathrm{CH}_{2} \mathrm{OH}\right), 62.5$ (CHAr), $108.6(\mathrm{C}-8), 110.0(\mathrm{C}-5), 122.3$ (C-4a), $126.0(\mathrm{C}-o), 127.4(\mathrm{C}-p), 127.8$ (C-p), 128.1 (C-m), 128.3 (C-o), 128.5 (C-8a), 128.8 (C-m), 136.7 (C-i), 143.0 (C-i), 147.8 (C-6), 148.0 (C-7), 171.9 (CO) ppm. HRMS calcd. for $\mathrm{C}_{25} \mathrm{H}_{25} \mathrm{NO}_{4}[\mathrm{M}+$ $\mathrm{H}]^{+}:$404.1862; found 404.1875.

\section{(1S)-1-(3,4-Dimethoxyphenyl)-2-[(1R)-2-hydroxy-1-}

\section{phenylethyl]-6,7-dimethoxy-3-0xo-1,2,3,4-}

tetrahydroisoquinoline (5d): Following the general procedure (reaction time $15 \mathrm{~min}$ ), from lactam 2 (150 $\mathrm{mg}, 0.46 \mathrm{mmol})$ in THF $(10 \mathrm{~mL})$ and 3,4-dimethoxyphenylmagnesium bromide $(0.5 \mathrm{M}$ in THF, $2.8 \mathrm{~mL}, 1.4 \mathrm{mmol})$ a residue was obtained. Flash chromatography $\left(1: 1 \quad \mathrm{Et}_{2} \mathrm{O}-\mathrm{EtOAc}\right.$, increasing polarity and 9:1 EtOAc-EtOH) gave 5d (115 mg, 54\%) as a yellow foam and 3 (50 $\mathrm{mg}, 33 \%)$. 5d: $[\alpha]^{22}=+17.1\left(c=1.0, \mathrm{CHCl}_{3}\right)$. IR $(\mathrm{KBr})$ : $v=1634,3406 \mathrm{~cm}^{-1} .{ }^{1} \mathrm{H}$ NMR $\left(400 \mathrm{MHz}, \mathrm{CDCl}_{3}, 25^{\circ} \mathrm{C}\right): \delta=3.68$ (d, $J=20.0 \mathrm{~Hz}, 1 \mathrm{H}, \mathrm{H}-4), 3.73$ (s, $\left.3 \mathrm{H}, \mathrm{OCH}_{3}\right), 3.79$ (s, $\left.3 \mathrm{H}, \mathrm{OCH}_{3}\right)$, $3.83\left(\mathrm{~s}, 3 \mathrm{H}, \mathrm{CH}_{3} \mathrm{O}\right), 3.86\left(\mathrm{~s}, 3 \mathrm{H}, \mathrm{CH}_{3} \mathrm{O}\right), 3.87(\mathrm{~d}, J=20.0 \mathrm{~Hz}, 1 \mathrm{H}$, $\mathrm{H}-4), 3.95$ (dd, $\left.J=11.6,8.4 \mathrm{~Hz}, 1 \mathrm{H}, \mathrm{CH}_{2} \mathrm{OH}\right), 4.01$ (dd, $J=11.6$, $\left.5.2 \mathrm{~Hz}, 1 \mathrm{H}, \mathrm{CH}_{2} \mathrm{OH}\right), 5.18$ (s, $\left.1 \mathrm{H}, \mathrm{H}-1\right), 5.93$ (dd, $J=8.4,5.2 \mathrm{~Hz}$, 1H, CHAr), 6.37 (s, 1H, H-2'), 6.58 (s, 1H, H-5'), 6.72 (s, 1H, H6'), 6.75 (s, 1H, H-8), 6.76 (s, 1H, H-5), 7.12-7.15 (m, 2H, ArH), 7.26-7.32 (m, 3H, ArH) ppm. ${ }^{13} \mathrm{C}$ NMR (100.6 MHz, $\mathrm{CDCl}_{3}$, $\left.25^{\circ} \mathrm{C}\right): \delta=37.4(\mathrm{C}-4), 55.8\left(\mathrm{OCH}_{3}\right), 55.9\left(\mathrm{OCH}_{3}\right), 55.9\left(\mathrm{OCH}_{3}\right)$, $56.0\left(\mathrm{OCH}_{3}\right), 60.4(\mathrm{C}-1), 62.5(\mathrm{CHAr}), 62.6\left(\mathrm{CH}_{2} \mathrm{OH}\right), 108.1(\mathrm{C}-8)$, 109.5 (C-5), 110.1 (C-2'), 111.3 (C-5'), 118.3 (C-6'), 122.4 (C-4a), 127.9 (C-p), 128.4 (C-o), 128.6 (C-8a), 128.6 (C-m), 134.4 (C-1'), 136.2 (C-i), 147.9 (C-6), 148.5 (C-4'), 148.6 (C-7), 149.2 (C-3'), 171.6 (CO) ppm. HRMS calcd. for $\mathrm{C}_{27} \mathrm{H}_{29} \mathrm{NO}_{6}[\mathrm{M}+\mathrm{H}]^{+}$: 464.2073; found 464.2081. $\mathrm{C}_{27} \mathrm{H}_{29} \mathrm{NO}_{6} .1 / 4 \mathrm{CHCl}_{3}$ (493.37): calcd. C 66.34, H 5.98, N 2.84; found C 66.65, H 6.33, N 2.49.

(1S)-2-[(1R)-2-Hydroxy-1-phenylethyl)]-6,7-dimethoxy-3-0xo-1(3,4,5-trimethoxyphenyl)-1,2,3,4-tetrahydroisoquinoline (5e): Following the general procedure (reaction time $20 \mathrm{~min}$ ), from lactam $2(500 \mathrm{mg}, 1.54 \mathrm{mmol})$ in THF $(10 \mathrm{~mL})$ and 3,4,5trimethoxyphenylmagnesium bromide $(0.5 \mathrm{M}$ in THF, $9.2 \mathrm{~mL}, 4.61$ $\mathrm{mmol})$ a residue was obtained. Flash chromatography $\left(\mathrm{Et}_{2} \mathrm{O}-\right.$ EtOAc, increasing polarity and 9:1 EtOAc-EtOH) gave 5e (370 $\mathrm{mg}, 49 \%)$ as a yellow foam and $3(135 \mathrm{mg}, 27 \%) . \mathbf{5 e}:[\alpha]^{22}{ }_{\mathrm{D}}=+$ $13.1\left(c=1.0, \mathrm{CHCl}_{3}\right)$. IR $(\mathrm{KBr}): \mathrm{v}=1684,2930 \mathrm{~cm}^{-1} .{ }^{1} \mathrm{H}$ NMR (300 MHz, $\left.\mathrm{CDCl}_{3}, 25^{\circ} \mathrm{C}\right): \delta=3.65(\mathrm{~d}, J=19.5 \mathrm{~Hz}, 1 \mathrm{H}, \mathrm{H}-4), 3.75$ $\left(\mathrm{s}, 3 \mathrm{H}, \mathrm{OCH}_{3}\right), 3.77\left(\mathrm{~s}, 3 \mathrm{H}, \mathrm{OCH}_{3}\right), 3.79\left(\mathrm{~s}, 3 \mathrm{H}, \mathrm{OCH}_{3}\right), 3.80$ (masked d, H-4), 3.81 (s, 3H, $\left.\mathrm{OCH}_{3}\right), 3.85$ (s, 3H, $\left.\mathrm{OCH}_{3}\right), 5.17$ (s, $1 \mathrm{H}, \mathrm{H}-1), 3.98-4.08$ (m, 2H, $\left.\mathrm{CH}_{2} \mathrm{OH}\right), 5.89$ (dd, $J=8.7,5.1 \mathrm{~Hz}, 1 \mathrm{H}$, CHAr), 6.40 (s, 1H, H-2'), 6.46 (s, 2H, H-5, H-8), 6.62 (s, 2H, H6'), 7.13-7.27 (m, 2H, ArH), 7.29-7.31 (m, 3H, ArH) ppm. ${ }^{13} \mathrm{C}$ NMR $\left(100.6 \mathrm{MHz}, \mathrm{CDCl}_{3}, 25^{\circ} \mathrm{C}\right): \delta=37.3(\mathrm{C}-4), 55.8\left(\mathrm{OCH}_{3}\right)$, $55.9\left(\mathrm{OCH}_{3}\right), 56.0\left(\mathrm{OCH}_{3}\right), 56.1\left(\mathrm{OCH}_{3}\right), 56.2\left(\mathrm{OCH}_{3}\right), 60.8(\mathrm{C}-1)$, $62.8\left(\mathrm{CH}_{2} \mathrm{OH}\right), 63.0$ (CHAr), 103.3 (C-2', C-6'), 108.1 (C-8), 110.0 (C-5), 122.7 (C-4a), 128.1 (C-p), 128.4 (C-o), 128.6 (C-8a), 128.6 (C-m), 136.1 (C-1'), 137.0 (C-i), 137.4 (C-4'), 147.9 (C-6), 148.6 (C-7), 153.4 (C-5', C-3'), 172.0 (CO) ppm. HRMS calcd. for
$\mathrm{C}_{28} \mathrm{H}_{31} \mathrm{NO}_{7}[\mathrm{M}+\mathrm{H}]^{+}:$494.2179; found 494.2183. $\mathrm{C}_{28} \mathrm{H}_{31} \mathrm{NO}_{7} .1 / 4$ $\mathrm{CHCl}_{3}$ (523.40): calcd. C 64.83, H 6.02, N 2.68; found C 64.69, H $6.26, \mathrm{~N} 2.42$.

$(1 S)$-[(R)-2-Hydroxy-1-phenylethyl]-6,7-dimethoxy-1-(pmethoxybenzyl)-3-0xo-1,2,3,4-tetrahydroisoquinoline (5f): Following the general procedure (reaction time $15 \mathrm{~min}$ ), from lactam 2 (150 mg, $0.46 \mathrm{mmol})$ in THF $(10 \mathrm{~mL})$ and 4methoxybenzylmagnesium chloride $(0.25 \mathrm{M}$ in THF, $5.5 \mathrm{~mL}, 1.4$ mmol) a residue was obtained. Flash chromatography (3:7 hexaneEtOAc, increasing polarity and 9:1 EtOAc-EtOH) gave $\mathbf{5 f}(129 \mathrm{mg}$, $63 \%)$ as a white foam and $\mathbf{3}(37 \mathrm{mg}, 25 \%) . \mathbf{5 f}:[\alpha]^{22}{ }_{\mathrm{D}}=+41.5(c=$ 1.0, $\left.\mathrm{CHCl}_{3}\right)$. IR (KBr): $v=1630,3393 \mathrm{~cm}^{-1} .{ }^{1} \mathrm{H}$ NMR (400 MHz, $\left.\mathrm{CDCl}_{3}, 25^{\circ} \mathrm{C}\right): \delta=2.84\left(\mathrm{dd}, J=13.1,8.5 \mathrm{~Hz}, 1 \mathrm{H}, \mathrm{CHCH}_{2} \mathrm{Ar}\right), 3.06$ (dd, $J=13.1,3.4 \mathrm{~Hz}, 1 \mathrm{H}, \mathrm{CHCH}_{2} \mathrm{Ar}$ ), 3.10 (d, $J=19.4 \mathrm{~Hz}, 1 \mathrm{H}, \mathrm{H}-$ 4), 3.39 (d, $J=19.4 \mathrm{~Hz}, 1 \mathrm{H}, \mathrm{H}-4), 3.51$ (s, $3 \mathrm{H}, \mathrm{OCH}_{3} \mathrm{C} 4$ '), 3.69 (s, $\left.3 \mathrm{H}, \mathrm{OCH}_{3}\right), 3.82\left(\mathrm{~s}, 3 \mathrm{H}, \mathrm{OCH}_{3}\right), 4.29(\mathrm{dd}, J=8.5,3.4 \mathrm{~Hz}, 1 \mathrm{H}, \mathrm{H}-$ 1), 4.35-4.44 (m, 2H, $\left.\mathrm{CH}_{2} \mathrm{OH}\right), 5.80$ (s, $1 \mathrm{H}, \mathrm{H}-5$ or H-8), 5.85 (t, $J$ $=6.5 \mathrm{~Hz}, 1 \mathrm{H}, \mathrm{CHAr}), 6.53(\mathrm{~s}, 1 \mathrm{H}, \mathrm{H}-5$ or H-8), $6.62(\mathrm{~s}, 4 \mathrm{H}, \mathrm{H}-2$ ', H-3', H-5', H-6'), 7.25-7.35 (m, 5H, ArH) ppm. ${ }^{13} \mathrm{C}$ NMR (75.4 $\left.\mathrm{MHz}, \mathrm{CDCl}_{3}, 25^{\circ} \mathrm{C}\right): \delta=37.2(\mathrm{C}-4), 41.9\left(\mathrm{ClCH}_{2}\right), 55.2\left(\mathrm{OCH}_{3}\right)$, $55.8\left(\mathrm{OCH}_{3}\right), 55.9\left(\mathrm{OCH}_{3}\right), 61.4(\mathrm{C}-1), 61.6(\mathrm{CHAr}), 63.6$ $\left(\mathrm{CH}_{2} \mathrm{OH}\right), 109.2$ (C-8), 109.7 (C-5), 113.5 (C-2', C-6'), 124.2 (C4a), 127.2 (C-8a), 127.9 (C-o), 128.0 (C- $p), 128.8$ (C-m), 131.1 (Cm), 131.1 (C-3', C-5'), 136.9 (C-i), 146.8 (C-6), 148.2 (C-7), 158.5 (C-4'), 172.3 (CO) ppm. HRMS calcd. for $\mathrm{C}_{27} \mathrm{H}_{29} \mathrm{NO}_{5}[\mathrm{M}+\mathrm{H}]^{+}$: 448.2124; found 448.2130. $\mathrm{C}_{27} \mathrm{H}_{29} \mathrm{NO}_{5} .3 / 4 \mathrm{H}_{2} \mathrm{O}$ (461.04): calcd. C 70.34, H 6.67, N 3.04; found C 70.03, H 6.42, N 2.87.

(1S)-2-[(1R)-2-Hydroxy-1-phenylethyl]-6,7-dimethoxy-3-oxo-1phenethyl-1,2,3,4-tetrahydroisoquinoline $(5 \mathrm{~g})$ : Following the general procedure (reaction time $40 \mathrm{~min}$ ), from lactam $2(400 \mathrm{mg}$, $1.23 \mathrm{mmol})$ in THF $(10 \mathrm{~mL})$ and phenethylmagnesium chloride (1 $\mathrm{M}$ in THF, $3.69 \mathrm{~mL}, 3.69 \mathrm{mmol})$ a residue was obtained. Flash chromatography (8:2 $\mathrm{Et}_{2} \mathrm{O}-\mathrm{EtOAc}$, increasing polarity and 9:1 EtOAc-EtOH) gave $\mathbf{5 g}(353 \mathrm{mg}, 67 \%)$ as a white foam and $\mathbf{3}$ (68 $\mathrm{mg}, 17 \%) .5 \mathrm{~g}:[\alpha]^{22}{ }_{\mathrm{D}}=+72.9\left(c=1.0, \mathrm{CHCl}_{3}\right)$. IR $(\mathrm{KBr}): \mathrm{v}=1632$, $3399 \mathrm{~cm}^{-1}$. ${ }^{1} \mathrm{H}$ NMR $\left(400 \mathrm{MHz}, \mathrm{CDCl}_{3}, 25^{\circ} \mathrm{C}\right): \delta=1.92-2.07(\mathrm{~m}$, $\left.1 \mathrm{H}, \mathrm{CH}_{2}\right), 2.10-2.18\left(\mathrm{~m}, 1 \mathrm{H}, \mathrm{CH}_{2}\right), 2.25-2.36\left(\mathrm{~m}, 1 \mathrm{H}, \mathrm{CH}_{2} \mathrm{Ar}\right)$, 2.50-2.57 (m, 1H, $\mathrm{CH}_{2} \mathrm{Ar}$ ), 3.59 (d, $\left.J=18.8 \mathrm{~Hz}, 1 \mathrm{H}, \mathrm{H}-4\right), 3.78$ (s, $\left.3 \mathrm{H}, \mathrm{OCH}_{3}\right), 3.81(\mathrm{~d}, J=18.8 \mathrm{~Hz}, 1 \mathrm{H}, \mathrm{H}-4), 3.87\left(\mathrm{~s}, 3 \mathrm{H}, \mathrm{OCH}_{3}\right)$, 4.10 (dd, $J=9.2,2.8 \mathrm{~Hz}, 1 \mathrm{H}, \mathrm{H}-1)$, 4.18-4.23 (m, $\left.2 \mathrm{H}, \mathrm{CH}_{2} \mathrm{OH}\right)$, 5.59 (dd, $J=7.6,6.0 \mathrm{~Hz}, 1 \mathrm{H}, \mathrm{CHAr}), 6.34$ (s, 1H, H-8), 6.69 (s, $1 \mathrm{H}$, H-5), 7.01 (d, $J=13.2 \mathrm{~Hz}, 2 \mathrm{H}, \mathrm{C}-2$ ', C-6'), 7.15 (m, 1H, H-4'), 7.20 (br s, 2H, C-3', C-5'), 7.24-7.26 (m, 5H, ArH) ppm. ${ }^{13} \mathrm{C}$ NMR $\left(100.6 \mathrm{MHz}, \mathrm{CDCl}_{3}, 25^{\circ} \mathrm{C}\right): \delta=31.5\left(\mathrm{CH}_{2} \mathrm{Ar}\right), 37.6(\mathrm{C}-4), 37.7$ $\left(\mathrm{CH}_{2}\right), 56.0\left(\mathrm{OCH}_{3}\right), 56.1\left(\mathrm{OCH}_{3}\right), 59.4(\mathrm{C}-1), 61.7(\mathrm{CHAr}), 63.3$ $\left(\mathrm{CH}_{2} \mathrm{OH}\right), 109.1$ (C-8), 110.5 (C-5), 123.9 (C-4a), 126.1 (C-4'), 127.8 (C-p), 127.8 (C-m), 128.1 (C-o), 128.3 (C-8a), 128.5 (C-2', C-6'), 128.6 (C-3', C-5'), 136.7 (C-i), 140.6 (C-1'), 147.3 (C-6), 148.4 (C-7), 171.9 (CO) ppm. HRMS calcd. for $\mathrm{C}_{27} \mathrm{H}_{29} \mathrm{NO}_{4}[\mathrm{M}+$ $\mathrm{H}]^{+}$: 432.2175; found 432.2180. $\mathrm{C}_{27} \mathrm{H}_{29} \mathrm{NO}_{4} .3 / 4 \quad \mathrm{H}_{2} \mathrm{O}$ (445.04): calcd. C 72.87, H 6.91, N 3.15; found C 72.95, H 6.87, N 2.95.

(1S)-1-Allyl-2-[(1R)-2-hydroxy-1-phenylethyl]-6,7-dimethoxy-3oxo-1,2,3,4-tetrahydroisoquinoline (5h): Following the general procedure (reaction time $2 \mathrm{~h}$ ), from lactam $2(200 \mathrm{mg}, 0.6 \mathrm{mmol})$ in THF $(10 \mathrm{~mL})$ and allylmagnesium bromide $\left(1 \mathrm{M}^{\text {in }} \mathrm{Et}_{2} \mathrm{O}, 1.85 \mathrm{~mL}\right.$, $1.85 \mathrm{mmol})$ a yellow oil was obtained. Flash chromatography (95:5 tert-butyl methyl ether-EtOAc) gave $\mathbf{5 h}(92 \mathrm{mg}, 42 \%)$ as a white 
oil and 1-(2-allyl-2-hydroxy-4-pentenyl)-2-[1-(2-hydroxy-1 $R$ phenylethylamino)-3-butenyl]-4,5-dimethoxybenzene (10) (103 mg, $39 \%)$. 5h: $[\alpha]^{22}{ }_{\mathrm{D}}=+16.8\left(c=1.0, \mathrm{CHCl}_{3}\right)$. IR $(\mathrm{KBr}): \mathrm{v}=1637$, $3386 \mathrm{~cm}^{-1} .{ }^{1} \mathrm{H}$ NMR $\left(300 \mathrm{MHz}, \mathrm{CDCl}_{3}, 25^{\circ} \mathrm{C}\right): \delta=2.18-2.33(\mathrm{~m}$, $\left.1 \mathrm{H}, \mathrm{CH}_{2} \mathrm{CH}=\right), 2.39-2.60\left(\mathrm{~m}, 1 \mathrm{H}, \mathrm{CH}_{2} \mathrm{CH}=\right), 3.54(\mathrm{~d}, J=19.5 \mathrm{~Hz}$, $1 \mathrm{H}, \mathrm{H}-4), 3.79$ (d, $J=19.5 \mathrm{~Hz}, 1 \mathrm{H}, \mathrm{H}-4), 3.76$ (s, $\left.3 \mathrm{H}, \mathrm{OCH}_{3}\right), 3.86$ (s, $\left.3 \mathrm{H}, \mathrm{OCH}_{3}\right), 4.26(\mathrm{~m}, 1 \mathrm{H}, \mathrm{H}-1), 4.28(\mathrm{dd}, J=11.4,7.8 \mathrm{~Hz}, 1 \mathrm{H}$, $\left.\mathrm{CH}_{2} \mathrm{OH}\right), 4.30\left(\mathrm{dd}, J=11.4,5.7 \mathrm{~Hz}, 1 \mathrm{H}, \mathrm{CH}_{2} \mathrm{OH}\right), 4.90(\mathrm{dd}, J=$ $\left.10.5,1.8 \mathrm{~Hz}, 1 \mathrm{H}, \mathrm{CH}_{2}=\right), 4.99\left(\mathrm{td}, J=6.0,1.8 \mathrm{~Hz}, 1 \mathrm{H}, \mathrm{CH}_{2}=\right), 5.51$ $(\mathrm{m}, 1 \mathrm{H}, \mathrm{CH}=), 5.68(\mathrm{dd}, J=7.8,5.7 \mathrm{~Hz}, 1 \mathrm{H}, \mathrm{H}-1), 6.31(\mathrm{~s}, 1 \mathrm{H}, \mathrm{H}-$ 8), 6.65 (s, 1H, H-5), 7.22-7.38 (m, 5H, ArH) ppm. ${ }^{13} \mathrm{C}$ NMR $(75.4$ $\left.\mathrm{MHz}, \mathrm{CDCl}_{3}, 25^{\circ} \mathrm{C}\right): \delta=37.6(\mathrm{C}-4), 40.8\left(\mathrm{CH}_{2} \mathrm{CH}=\right), 55.8\left(\mathrm{OCH}_{3}\right)$, $56.0\left(\mathrm{OCH}_{3}\right), 60.1(\mathrm{C}-1), 61.3(\mathrm{CHAr}), 63.2\left(\mathrm{CH}_{2} \mathrm{OH}\right), 109.0(\mathrm{C}-8)$, $110.0(\mathrm{C}-5), 118.9\left(\mathrm{CH}_{2}=\right), 123.6(\mathrm{C}-4 \mathrm{a}), 127.6(\mathrm{C}-p), 127.7(\mathrm{C}-o)$, $128.4(\mathrm{C}-m), 128.6(\mathrm{C}-8 \mathrm{a}), 133.0(\mathrm{CH}=), 136.8(\mathrm{C}-i), 147.1(\mathrm{C}-7)$, 148.2 (C-6), 171.8 (CO) ppm. HRMS calcd. for $\mathrm{C}_{22} \mathrm{H}_{25} \mathrm{NO}_{4}[\mathrm{M}+$ $\mathrm{H}]^{+}: 368.1862$; found $368.1873 . \mathrm{C}_{22} \mathrm{H}_{25} \mathrm{NO}_{4} .1 / 2 \mathrm{H}_{2} \mathrm{O}$ (411.5): calcd. C 70.05, H 7.10, N 3.40; found C 66.69, H 6.70, N 3.55. 10: IR $(\mathrm{KBr}): \mathrm{v}=1638,3073,3324 \mathrm{~cm}^{-1} .{ }^{1} \mathrm{H}$ NMR $\left(400 \mathrm{MHz}, \mathrm{CDCl}_{3}\right.$, $\left.25^{\circ} \mathrm{C}\right): \delta=2.10-2.19\left(\mathrm{~m}, 4 \mathrm{H}, 2 \mathrm{CH}_{2} \mathrm{CHCH}_{2}\right), 2.38-2.53(\mathrm{~m}, 2 \mathrm{H}$, $\left.\mathrm{CHCH}_{2} \mathrm{CHCH}_{2}\right), 2.56\left(\mathrm{~d}, J=14.8 \mathrm{~Hz}, 1 \mathrm{H}, \mathrm{CH}_{2} \mathrm{Ar}\right), 2.66(\mathrm{~d}, J=$ $\left.14.8 \mathrm{~Hz}, 1 \mathrm{H}, \mathrm{CH}_{2} \mathrm{Ar}\right), 3.57\left(\mathrm{dd}, \mathrm{J}=10.8,8.0 \mathrm{~Hz}, 1 \mathrm{H}, \mathrm{CH}_{2} \mathrm{OH}\right)$, $3.67\left(\mathrm{dd}, J=10.8,4.4 \mathrm{~Hz}, 1 \mathrm{H}, \mathrm{CH}_{2} \mathrm{OH}\right), 3.85\left(\mathrm{~s}, 3 \mathrm{H}, \mathrm{OCH}_{3}\right), 3.86$ (dd, $J=8.0,4.4 \mathrm{~Hz}, 1 \mathrm{H}, \mathrm{CHAr}), 3.89\left(\mathrm{~s}, 3 \mathrm{H}, \mathrm{OCH}_{3}\right), 4.05(\mathrm{t}, J=$ $\left.6.4 \mathrm{~Hz}, 1 \mathrm{H}, \mathrm{CHCH}_{2} \mathrm{CHCH}_{2}\right), 4.98-5.09\left(\mathrm{~m}, 2 \mathrm{H}, \mathrm{CHCH}_{2} \mathrm{CHCH}_{2}\right)$, 5.10-5.15 (m, 4H, $\left.2 \mathrm{CH}_{2} \mathrm{CHCH}_{2}\right), \quad 5.64-5.72(\mathrm{~m}, \quad 1 \mathrm{H}$, $\left.\mathrm{CHCH}_{2} \mathrm{CHCH}_{2}\right)$, 5.74-5.84 (m, 2H, 2- $\left.\mathrm{CH}_{2} \mathrm{CHCH}_{2}\right), 6.65(\mathrm{~s}, 1 \mathrm{H}$, $\left.\mathrm{CH}_{3} \mathrm{OCCH}\right), 6.85\left(\mathrm{~s}, 1 \mathrm{H}, \mathrm{CH}_{3} \mathrm{OCCH}\right), 7.26-7.28(\mathrm{~m}, 5 \mathrm{H}, \mathrm{ArH})$ ppm. ${ }^{13} \mathrm{C} \quad \mathrm{NMR} \quad\left(75.4 \mathrm{MHz}, \mathrm{CDCl}_{3}, \quad 25^{\circ} \mathrm{C}\right): \quad \delta=40.3$ $\left(\mathrm{CHCH}_{2} \mathrm{CHCH}_{2}\right), \quad 40.4 \quad\left(\mathrm{CH}_{2} \mathrm{Ar}\right), \quad 43.7 \quad\left(\mathrm{CH}_{2} \mathrm{CHCH}_{2}\right), \quad 44.0$ $\left(\mathrm{CH}_{2} \mathrm{CHCH}_{2}\right), 55.1\left(\mathrm{CHCH}_{2} \mathrm{CHCH}_{2}\right), 55.6\left(\mathrm{OCH}_{3}\right), 55.7\left(\mathrm{OCH}_{3}\right)$, 61.5 (CHAr), $66.3\left(\mathrm{CH}_{2} \mathrm{OH}\right), 73.3(\mathrm{COH}), 110.1(\mathrm{C}-3), 114.8(\mathrm{C}-6)$, $117.3\left(\mathrm{CHCH}_{2} \mathrm{CHCH}_{2}\right), 118.5\left(\mathrm{CH}_{2} \mathrm{CHCH}_{2}\right), 118.6\left(\mathrm{CH}_{2} \mathrm{CHCH}_{2}\right)$, 127.0 (C-2), 127,3 (C-m), 127.4 (C- $p), 128.4$ (C-o), 133.8 $\left(\mathrm{CH}_{2} \mathrm{CHCH}_{2}\right), \quad 133.9 \quad\left(\mathrm{CH}_{2} \mathrm{CHCH}_{2}\right), \quad 135.0 \quad(\mathrm{C}-1), \quad 135.2$ $\left(\mathrm{CHCH}_{2} \mathrm{CHCH}_{2}\right), 140.9$ (C-i), 146.9 (C-4), 147.5 (C-5) ppm. HRMS calcd. for $\mathrm{C}_{28} \mathrm{H}_{37} \mathrm{NO}_{4}[\mathrm{M}+\mathrm{H}]^{+}$: 452.2801; found 452.2784. $\mathrm{C}_{28} \mathrm{H}_{37} \mathrm{NO}_{4} .1 / 2 \mathrm{H}_{2} \mathrm{O}(460.61)$ : calcd. C 73.01, H 8.32, N 3.04; found C 73.24, H 8.13, N 2.75 .

\section{(1S)-1-[2-(1,3-Dioxan-2-yl)ethyl]-2-[(1R)-2-hydroxy-1- phenylethyl]-6,7-dimethoxy-3-0xo-1,2,3,4-}

tetrahydroisoquinoline (5i): Following the general procedure (reaction time $2.5 \mathrm{~h})$, from lactam $2(400 \mathrm{mg}, 1.23 \mathrm{mmol})$ in THF $(20 \mathrm{~mL})$ and 2-(1,3-dioxan-2-yl)ethylmagnesium bromide $(0.5 \mathrm{M}$ in THF, $7.4 \mathrm{~mL}, 3.69 \mathrm{mmol})$ a yellow solid was obtained. Flash chromatography $\left(1: 1 \mathrm{Et}_{2} \mathrm{O}-\mathrm{EtOAc}\right.$, increasing polarity and 9:1 EtOAc-EtOH) gave $\mathbf{5 i}(246 \mathrm{mg}, 45 \%)$ as a yellow foam and $\mathbf{3}(121$ $\mathrm{mg}, 30 \%) .5 \mathbf{i}:[\alpha]^{22}{ }_{\mathrm{D}}=+38.5\left(c=1.0, \mathrm{CHCl}_{3}\right)$. IR $(\mathrm{KBr}): \mathrm{v}=1632$, $3399 \mathrm{~cm}^{-1}$. ${ }^{1} \mathrm{H}$ NMR $\left(400 \mathrm{MHz}, \mathrm{CDCl}_{3}, 25^{\circ} \mathrm{C}\right): \delta=1.31(\mathrm{dt}, J=$ 13.6, $\left.1.2 \mathrm{~Hz}, 2 \mathrm{H}, \mathrm{H}-1^{\prime}\right), 1.41-1.46\left(\mathrm{~m}, 2 \mathrm{H}, \mathrm{OCH}_{2} \mathrm{CH}_{2}\right), 1.72-1.81$ (m, 1H, H-2'), 1.92-2.06 (m, 1H, H-2'), 3.56 (d, $J=19.0 \mathrm{~Hz}, 1 \mathrm{H}$, $\mathrm{H}-4$ ), 3.68 (ddd, $J=15.2,12.0,3.6 \mathrm{~Hz}, 2 \mathrm{H}, \mathrm{CH}_{2} \mathrm{CH}_{2} \mathrm{O}$ ), 3.75 (s, $3 \mathrm{H}$, $\left.\mathrm{OCH}_{3}\right), 3.79$ (d, $\left.J=19.0 \mathrm{~Hz}, 1 \mathrm{H}, \mathrm{H}-4\right), 3.85\left(\mathrm{~s}, 3 \mathrm{H}, \mathrm{OCH}_{3}\right), 4.03$ $\left(\mathrm{m}, 2 \mathrm{H}, \mathrm{CH}_{2} \mathrm{CH}_{2} \mathrm{O}\right), 4.40\left(\mathrm{t}, J=4.8 \mathrm{~Hz}, 1 \mathrm{H}, \mathrm{CHO}_{2}\right), 4.15(\mathrm{dd}, J=$ 10.0, $3.2 \mathrm{~Hz}, 1 \mathrm{H}, \mathrm{H}-1), 4.23-4.32\left(\mathrm{~m}, 2 \mathrm{H}, \mathrm{CH}_{2} \mathrm{OH}\right), 5.76$ (dd, $J=$ 8.0, 5.6 Hz, 1H, CHAr), 6.34 (s, 1H, H-8), 6.65 (s, 1H, H-5), 7.16 $(\mathrm{d}, J=1.6 \mathrm{~Hz}, 1 \mathrm{H}, \mathrm{ArH}), 7.18(\mathrm{~d}, J=2.0 \mathrm{~Hz}, 1 \mathrm{H}, \mathrm{ArH}), 7.25-7.30$ (m, 3H, ArH) ppm. $\left.{ }^{13} \mathrm{C} \mathrm{NMR} \mathrm{(100.6} \mathrm{MHz,} \mathrm{CDCl}_{3}, 25^{\circ} \mathrm{C}\right): \delta=25.6$ (C-1'), 30.3 (C-2'), $31.1\left(\mathrm{CH}_{2} \mathrm{CH}_{2} \mathrm{O}\right), 37.6(\mathrm{C}-4), 55.9\left(\mathrm{OCH}_{3}\right)$,
$56.0\left(\mathrm{OCH}_{3}\right), 58.9$ (C-1), 60.3 (CHAr), $63.1\left(\mathrm{CH}_{2} \mathrm{OH}\right), 66.7$ $\left(2 \mathrm{CH}_{2} \mathrm{CH}_{2} \mathrm{O}\right), 101.6\left(\mathrm{CHO}_{2}\right), 109.4(\mathrm{C}-8), 110.4(\mathrm{C}-5), 123.8(\mathrm{C}-$ 4a), 127.7 (C- $p$ ), 127.8 (C-o), 128.1 (C-8a), 128.5 (C-m), 136.8 (Ci), 147.2 (C-6), 148.3 (C-7), 171.9 (CO) ppm. HRMS calcd. for $\mathrm{C}_{25} \mathrm{H}_{31} \mathrm{NO}_{6}[\mathrm{M}+\mathrm{H}]^{+}:$442.2223; found 442.2219.

General Procedure for $\mathrm{Na} / \mathrm{liq}$. $\mathrm{NH}_{3}$ Reaction: Into a threenecked round-bottomed equipped with a coldfinger condenser charged with dry ice-acetone was condensed $\mathrm{NH}_{3}$ at $-78{ }^{\circ} \mathrm{C}$. The temperature was raised to $-33{ }^{\circ} \mathrm{C}$, and a solution of lactam 5 in THF was added. Then, sodium metal was added in small portions until the blue color persisted. After the mixture was stirred at -33 ${ }^{\circ} \mathrm{C}$ for $1 \mathrm{~min}$, the reaction was quenched by the addition of solid $\mathrm{NH}_{4} \mathrm{Cl}$ until the blue color disappeared. The mixture was stirred at $\mathrm{rt}$ for $4 \mathrm{~h}$, poured into water, and extracted with $\mathrm{Et}_{2} \mathrm{O}$. The combined organic extracts were dried and concentrated to give a residue, which was chromatographed.

\section{(1S)-6,7-Dimethoxy-1-methyl-3-oxo-1,2,3,4-}

tetrahydroisoquinoline (6a): Operating as described in the general procedure, from 5a $(200 \mathrm{mg}, 0.59 \mathrm{mmol})$ in THF $(7 \mathrm{~mL})$ and $\mathrm{NH}_{3}(50 \mathrm{~mL})$ a clear marron residue was obtained. Flash chromatography (9:1 EtOAc-EtOH) afforded $\mathbf{6 a}(110 \mathrm{mg}, 85 \%)$ as a white solid. $[\alpha]^{22}{ }_{\mathrm{D}}=+10.0\left(c=1.0, \mathrm{CHCl}_{3}\right)$. IR $(\mathrm{KBr}): \nu=1668$, $3217 \mathrm{~cm}^{-1}$. ${ }^{1} \mathrm{H}$ NMR $\left(400 \mathrm{MHz}, \mathrm{CDCl}_{3}, 25^{\circ} \mathrm{C}\right): \delta=1.51(\mathrm{~d}, J=6.4$ $\left.\mathrm{Hz}, 3 \mathrm{H}, \mathrm{CH}_{3}\right), 3.48$ (d, $\left.J=20.0 \mathrm{~Hz}, 1 \mathrm{H}, \mathrm{H}-4\right), 3.60(\mathrm{~d}, \mathrm{~J}=20.0 \mathrm{~Hz}$, $1 \mathrm{H}, \mathrm{H}-4), 3.87$ (s, $\left.6 \mathrm{H}, 2 \mathrm{OCH}_{3}\right), 4.60(\mathrm{~m}, 1 \mathrm{H}, \mathrm{H}-1), 6.60$ (s, 1H, H8), 6.61 (s, 1H, H-5), 6.90 (br s, 1H, NH) ppm. ${ }^{13} \mathrm{C}$ NMR (100.6 $\left.\mathrm{MHz}, \mathrm{CDCl}_{3}, 25^{\circ} \mathrm{C}\right): \delta=24.4\left(\mathrm{CH}_{3}\right), 35.4(\mathrm{C}-4), 51.3(\mathrm{C}-1), 56.0$ $\left(\mathrm{OCH}_{3}\right), 56.1\left(\mathrm{OCH}_{3}\right), 108.2(\mathrm{C}-8), 110.4(\mathrm{C}-5), 122.8(\mathrm{C}-4 \mathrm{a})$, 127.8 (C-8a), 148.0 (C-6), 148.4 (C-7), 171.5 (CO) ppm. HRMS calcd. for $\mathrm{C}_{12} \mathrm{H}_{15} \mathrm{NO}_{3}[\mathrm{M}+\mathrm{H}]^{+}$: 222.1124; found 222.1122 . $\mathrm{C}_{12} \mathrm{H}_{15} \mathrm{NO}_{3}$ (221.25): calcd. C 65.14, H 6.83, N 6.33; found $\mathrm{C}$ 64.89, H 6.76, N 6.16 .

\section{(1S)-1-Ethyl-6,7-dimethoxy-3-0xo-1,2,3,4-}

tetrahydroisoquinoline (6b): Operating as described in the general procedure, from $\mathbf{5 b}(230 \mathrm{mg}, 0.65 \mathrm{mmol})$ in THF $(4 \mathrm{~mL})$ and $\mathrm{NH}_{3}(30 \mathrm{~mL})$ a residue was obtained. Flash chromatography (EtOAc) afforded $\mathbf{6 b}(140 \mathrm{mg}, 92 \%)$ as a white oil. $[\alpha]^{22}{ }_{\mathrm{D}}=+21.7$ $\left(c=0.57, \mathrm{CHCl}_{3}\right)$. IR $(\mathrm{KBr}): \mathrm{v}=1654,2972 \mathrm{~cm}^{-1} .{ }^{1} \mathrm{H}$ NMR $(400$ $\left.\mathrm{MHz}, \mathrm{CDCl}_{3}, 25^{\circ} \mathrm{C}\right): \delta=0.92\left(\mathrm{t}, J=8.0 \mathrm{~Hz}, 3 \mathrm{H}, \mathrm{CH}_{3}\right), 1.81(\mathrm{~m}, 2 \mathrm{H}$, $\left.\mathrm{CH}_{2}\right), 3.46(\mathrm{~d}, J=20.0 \mathrm{~Hz}, 1 \mathrm{H}, \mathrm{H}-4), 3.62$ (d, $J=20.0 \mathrm{~Hz}, 1 \mathrm{H}, \mathrm{H}-$ 4), 3.87 (s, 6H, 2OCH $\mathrm{OCH}_{3}, 4.44$ (m, 1H, H-1), 6.60 (s, 1H, H-8), 6.62 (s, 1H, H-5), 7.70 (br s, $1 \mathrm{H}, \mathrm{NH}) \mathrm{ppm} .{ }^{13} \mathrm{C} \mathrm{NMR}(100.6 \mathrm{MHz}$, $\left.\mathrm{CDCl}_{3}, 25^{\circ} \mathrm{C}\right): \delta=9.1\left(\mathrm{CH}_{3}\right), 31.7\left(\mathrm{CH}_{2}\right), 35.2(\mathrm{C}-4), 55.9\left(\mathrm{OCH}_{3}\right)$, $56.0\left(\mathrm{OCH}_{3}\right), 57.2(\mathrm{C}-1), 108.8(\mathrm{C}-8), 110.3(\mathrm{C}-5), 123.2(\mathrm{C}-4 \mathrm{a})$, 126.2 (C-8a), 147.8 (C-7), 148.4 (C-6), 171.9 (CO) ppm. HRMS calcd. for $\mathrm{C}_{13} \mathrm{H}_{17} \mathrm{NO}_{3}[\mathrm{M}+\mathrm{H}]^{+}$: 236.1286; found 236.1280.

(1S)-1-[3,4-(Dimethoxyphenyl)]-6,7-dimethoxy-3-oxo-1,2,3,4tetrahydroisoquinoline (6d): Operating as described in the general procedure, from $\mathbf{5 d}(215 \mathrm{mg}, 0.46 \mathrm{mmol})$ in THF $(3 \mathrm{~mL})$ and $\mathrm{NH}_{3}(35 \mathrm{~mL})$ a residue was obtained. Flash chromatography (2:8 to $1: 9$ hexane-EtOAc) afforded $\mathbf{6 d} 121 \mathrm{mg}, 77 \%$ ) as a yellow foam. IR (KBr): $v=1647,2920 \mathrm{~cm}^{-1} .{ }^{1} \mathrm{H}$ NMR (400 MHz, $\mathrm{CDCl}_{3}$, $\left.25^{\circ} \mathrm{C}\right): \delta=3.52(\mathrm{~d}, J=24.0 \mathrm{~Hz}, 1 \mathrm{H}, \mathrm{H}-4), 3.66(\mathrm{~d}, J=24.0 \mathrm{~Hz}, 1 \mathrm{H}$, $\mathrm{H}-4), 3.71\left(\mathrm{~s}, 3 \mathrm{H}, \mathrm{OCH}_{3}\right), 3.82\left(\mathrm{~s}, 3 \mathrm{H}, \mathrm{OCH}_{3}\right), 3.86\left(\mathrm{~s}, 3 \mathrm{H}, \mathrm{OCH}_{3}\right)$, 3.89 (s, 3H, $\left.\mathrm{OCH}_{3}\right), 5.54$ (s, 1H, H-1), 6.37 (s, 1H, H-2'), 6.65 (s, 1H, H-5'), 6.72 (s, 1H, H-6'), 6.83 (s, 1H, H-8), 6.84 (s, 1H, H-5), 
7.05 (br s, $1 \mathrm{H}, \mathrm{NH}) \mathrm{ppm} .{ }^{13} \mathrm{C} \mathrm{NMR}\left(100.6 \mathrm{MHz}, \mathrm{CDCl}_{3}, 25^{\circ} \mathrm{C}\right): \delta$ $=35.5(\mathrm{C}-4), 55.6\left(\mathrm{OCH}_{3}\right), 55.7\left(\mathrm{OCH}_{3}\right), 55.8\left(\mathrm{OCH}_{3}\right), 59.6(\mathrm{C}-1)$, 109.5 (C-8), 110.0 (C-5), 110.1 (C-2'), 110.9 (C-5'), 119.7 (C-6'), 123.0 (C-4a), 126.1 (C-8a), 134.2 (C-1'), 147.7 (C-6), 148.5 (C-4'), 148.8 (C-7), 149.3 (C-3'), 170.8 (CO) ppm. HRMS calcd. for $\mathrm{C}_{19} \mathrm{H}_{21} \mathrm{NO}_{5}[\mathrm{M}+\mathrm{H}]^{+}:$344.1498; found 344.1491.

(1S)-6,7-Dimethoxy-3-0xo-1-[3,4,5-(trimethoxyphenyl)]-1,2,3,4tetrahydroisoquinoline (6e): Operating as described in the general procedure, from 5 e $(150 \mathrm{mg}, 0.30 \mathrm{mmol})$ in THF $(2 \mathrm{~mL})$ and $\mathrm{NH}_{3}(30 \mathrm{~mL})$ a residue was obtained. Flash chromatography (EtOAc) afforded $\mathbf{6 e}(98 \mathrm{mg}, 87 \%)$ as a yellow foam. IR (KBr): $v=1663,2926 \mathrm{~cm}^{-1} .{ }^{1} \mathrm{H}$ NMR $\left(300 \mathrm{MHz}, \mathrm{CDCl}_{3}, 25^{\circ} \mathrm{C}\right): \delta=3.56$ (d, $J=18.0 \mathrm{~Hz}, 1 \mathrm{H}, \mathrm{H}-4), 3.71$ (d, $J=18.0 \mathrm{~Hz}, 1 \mathrm{H}, \mathrm{H}-4), 3.72$ (s, $\left.3 \mathrm{H}, \mathrm{OCH}_{3}\right), 3.76\left(\mathrm{~s}, 3 \mathrm{H}, \mathrm{OCH}_{3}\right), 3.79\left(\mathrm{~s}, 3 \mathrm{H}, \mathrm{OCH}_{3}\right), 3.83(\mathrm{~s}, 3 \mathrm{H}$, $\left.\mathrm{OCH}_{3}\right), 3.84\left(\mathrm{~s}, 3 \mathrm{H}, \mathrm{OCH}_{3}\right), 5.25$ (s, $\left.1 \mathrm{H}, \mathrm{H}-1\right), 6.41(\mathrm{~s}, 2 \mathrm{H}, \mathrm{H}-2$ ', H-6'), 6.66 (s, 1H, H-8), 6.73 (s, 1H, H-5), 7.25 (br s, 1H, NH) ppm. ${ }^{13} \mathrm{C}$ NMR $\left(75.4 \mathrm{MHz}, \mathrm{CDCl}_{3}, 25^{\circ} \mathrm{C}\right): \delta=38.8(\mathrm{C}-4), 55.8$ $\left(\mathrm{OCH}_{3}\right), 55.9\left(\mathrm{OCH}_{3}\right), 56.0\left(\mathrm{OCH}_{3}\right), 56.1\left(\mathrm{OCH}_{3}\right), 60.0(\mathrm{C}-1)$, $60.1\left(\mathrm{OCH}_{3}\right), 104.3$ (C-8), 105.4 (C-2', C-6'), 113.9 (C-5), 125.4 (C-4a), 131.2 (C-4'), 131.3 (C-8a), 133.4 (C-1'), 147.7 (C-6), 148.1 (C-7), 153.1 (C-3', C-5'), 173.7 (CO) ppm. HRMS calcd. for $\mathrm{C}_{20} \mathrm{H}_{23} \mathrm{NO}_{6}[\mathrm{M}+\mathrm{H}]^{+}: 374.1603$; found 374.1592 .

\section{(1S)-6,7-Dimethoxy-1-(p-methoxybenzyl)-3-oxo-1,2,3,4-}

tetrahydroisoquinoline (6f): Operating as described in the general procedure, from $\mathbf{5 f}(95 \mathrm{mg}, 0.21 \mathrm{mmol})$ in THF $(2 \mathrm{~mL})$ and $\mathrm{NH}_{3}$ $(30 \mathrm{~mL})$ a residue was obtained. Flash chromatography (EtOAc to 95:5 EtOAc-EtOH) afforded $\mathbf{6 f}(58 \mathrm{mg}, 85 \%)$ as a yellow foam. $[\alpha]^{22}{ }_{\mathrm{D}}=-61.2\left(c=0.5, \mathrm{CHCl}_{3}\right)$. IR $(\mathrm{KBr}): \mathrm{v}=1630,2934 \mathrm{~cm}^{-1}$. ${ }^{1} \mathrm{H}$ NMR $\left(400 \mathrm{MHz}, \mathrm{CDCl}_{3}, 25^{\circ} \mathrm{C}\right): \delta=2.87(\mathrm{~d}, J=20.0 \mathrm{~Hz}, 1 \mathrm{H}$, H-4), 2.91 (dd, $J=13.5,6.0 \mathrm{~Hz}, 1 \mathrm{H}, \mathrm{CH}_{2}$ ), 3.03 (dd, $J=13.5,4.0$ $\left.\mathrm{Hz}, 1 \mathrm{H}, \mathrm{CH}_{2}\right), 3.23$ (d, $\left.J=20.0 \mathrm{~Hz}, 1 \mathrm{H}, \mathrm{H}-4\right), 3.77$ (s, $1 \mathrm{H}, \mathrm{OCH}_{3}$ ), $3.83\left(\mathrm{~s}, 1 \mathrm{H}, \mathrm{OCH}_{3}\right), 3.86\left(\mathrm{~s}, 1 \mathrm{H}, \mathrm{OCH}_{3}\right), 4.68(\mathrm{~m}, 1 \mathrm{H}, \mathrm{H}-1), 6.49(\mathrm{~s}$ $1 \mathrm{H}, \mathrm{H}-8), 6.55$ (s, 1H, H-5), 6.76 (d, $J=8.2 \mathrm{~Hz}, 2 \mathrm{H}, \mathrm{H}-2$ '), 6.86 (d, $J=8.2 \mathrm{~Hz}, 2 \mathrm{H}, \mathrm{H}-3$ '), 6.95 (br s, $1 \mathrm{H}, \mathrm{NH}) \mathrm{ppm} .{ }^{13} \mathrm{C}$ NMR $(100.6$ $\left.\mathrm{MHz}, \mathrm{CDCl}_{3}, 25^{\circ} \mathrm{C}\right): \delta=35.0(\mathrm{C}-4), 44.5\left(\mathrm{CH}_{2}\right), 55.2\left(\mathrm{OCH}_{3}\right), 55.9$ $(\mathrm{C}-1), 56.0\left(\mathrm{OCH}_{3}\right), 57.3\left(\mathrm{OCH}_{3}\right), 108.9(\mathrm{C}-8), 110.1(\mathrm{C}-5), 113.8$ (C-3'), 123.9 (C-4a), 125.2 (C-2'), 127.8 (C-8a), 131.8 (C-1'), 147.7 (C-6), 148.4 (C-7), 158.5 (C-4'), 172.3 (CO) ppm. HRMS calcd. for $\mathrm{C}_{19} \mathrm{H}_{21} \mathrm{NO}_{4}[\mathrm{M}+\mathrm{H}]^{+}$: 328.1549; found 328.1532 .

\section{(1S)-6,7-Dimethoxy-3-oxo-1-phenethyl-1,2,3,4-}

tetrahydroisoquinoline (6g): Operating as described in the general procedure, from $\mathbf{5 g}(100 \mathrm{mg}, 0.23 \mathrm{mmol})$ in THF $(2 \mathrm{~mL})$ and $\mathrm{NH}_{3}(25 \mathrm{~mL})$ a residue was obtained. Flash chromatography (EtOAc) afforded $\mathbf{6 g}(56 \mathrm{mg}, 79 \%)$ as a white foam. $[\alpha]^{22}{ }_{\mathrm{D}}=+16.0$ $\left(c=1.0, \mathrm{CHCl}_{3}\right)$. IR (KBr): $\mathrm{v}=1674 \mathrm{~cm}^{-1}$. ${ }^{1} \mathrm{H} \mathrm{NMR}(400 \mathrm{MHz}$, $\left.\mathrm{CDCl}_{3}, 25^{\circ} \mathrm{C}\right): \delta=2.03-2.09\left(\mathrm{~m}, 2 \mathrm{H}, \mathrm{CH}_{2}\right), 2.62-2.70(\mathrm{~m}, 2 \mathrm{H}$, $\left.\mathrm{CH}_{2} \mathrm{Ar}\right), 3.48$ (d, $\left.J=20.0 \mathrm{~Hz}, 1 \mathrm{H}, \mathrm{H}-4\right), 3.64$ (d, $J=20.0 \mathrm{~Hz}, 1 \mathrm{H}$, $\mathrm{H}-4), 3.86$ (s, 3H, $\left.\mathrm{OCH}_{3}\right), 3.87$ (s, 3H, $\left.\mathrm{OCH}_{3}\right), 4.60(\mathrm{~m}, 1 \mathrm{H}, \mathrm{H}-1)$, 6.60 (s, 2H, H-5, H-8), 7.14-7.19 (m, 3H, ArH), 7.25-7.28 (m, 2H, $\mathrm{ArH}), 7.43(\mathrm{~m}, 1 \mathrm{H}, \mathrm{NH})$ ppm. ${ }^{13} \mathrm{C} \mathrm{NMR}\left(100.6 \mathrm{MHz}, \mathrm{CDCl}_{3}\right.$, $\left.25^{\circ} \mathrm{C}\right): \delta=31.2\left(\mathrm{CH}_{2} \mathrm{Ar}\right), 35.4(\mathrm{C}-4), 40.5\left(\mathrm{CH}_{2}\right), 55.7(\mathrm{C}-1), 56.0$ $\left(\mathrm{OCH}_{3}\right), 56.1\left(\mathrm{OCH}_{3}\right), 108.7(\mathrm{C}-8), 110.5(\mathrm{C}-5), 123.2(\mathrm{C}-4 \mathrm{a})$, 126.1 (C-8a), 126.3 (C-4'), 128.3 (C-3'), 128.5 (C-2'), 140.9 (C1'), 147.9 (C-6), 148.5 (C-7), 171.7 (CO) ppm. HRMS calcd. for $\mathrm{C}_{19} \mathrm{H}_{21} \mathrm{NO}_{3}[\mathrm{M}+\mathrm{H}]^{+}:$312.1599; found 312.1598.
(1S)-1-Allyl-6,7-dimethoxy-3-0x0-1,2,3,4-

tetrahydroisoquinoline (6h): Operating as described in the general procedure, from $\mathbf{5 h}(80 \mathrm{mg}, 0.22 \mathrm{mmol})$ in THF $(2 \mathrm{~mL})$ and $\mathrm{NH}_{3}(25 \mathrm{~mL})$ a residue was obtained. Flash chromatography (EtOAc) afforded $\mathbf{6 h}(48.3 \mathrm{mg}, 90 \%)$ as a yellow foam. $[\alpha]^{22}{ }_{\mathrm{D}}=-$ $10.5\left(c=0.6, \mathrm{CHCl}_{3}\right)$. IR $(\mathrm{KBr}): \mathrm{v}=1667,2918 \mathrm{~cm}^{-1} .{ }^{1} \mathrm{H}$ NMR $\left(400 \mathrm{MHz}, \mathrm{CDCl}_{3}, 25^{\circ} \mathrm{C}\right): \delta=2.45(\mathrm{ddd}, J=14.0,7.2,7.2 \mathrm{~Hz}, 1 \mathrm{H}$, $\left.\mathrm{CH}_{2}\right), 2.57-2.61\left(\mathrm{~m}, 1 \mathrm{H}, \mathrm{CH}_{2}\right), 3.47(\mathrm{~d}, J=20.0 \mathrm{~Hz}, 1 \mathrm{H}, \mathrm{H}-4), 3.60$ $(\mathrm{d}, J=20.0 \mathrm{~Hz}, 1 \mathrm{H}, \mathrm{H}-4), 3.88\left(\mathrm{~s}, 6 \mathrm{H}, 2 \mathrm{OCH}_{3}\right), 4.52(\mathrm{~m}, 1 \mathrm{H}, \mathrm{H}-1)$, 5.12-5.17 (m, 2H, $\left.=\mathrm{CH}_{2}\right), 5.69-5.79(\mathrm{~m}, 1 \mathrm{H}, \mathrm{CH}=), 6.60(\mathrm{~s}, 1 \mathrm{H}, \mathrm{H}-$ 8), 6.65 (s, 1H, H-5), 6.80 (br s, $1 \mathrm{H}, \mathrm{NH}) \mathrm{ppm} .{ }^{13} \mathrm{C}$ NMR $(100.6$ $\left.\mathrm{MHz}, \mathrm{CDCl}_{3}, 25^{\circ} \mathrm{C}\right): \delta=35.4(\mathrm{C}-4), 43.2\left(\mathrm{CH}_{2}\right), 55.5(\mathrm{C}-1), 55.9$ $\left(\mathrm{OCH}_{3}\right), 56.0\left(\mathrm{OCH}_{3}\right), 108.6(\mathrm{C}-8), 110.4(\mathrm{C}-5), 119.9\left(=\mathrm{CH}_{2}\right)$, 123.3 (C-4a), 125.6 (C-8a), 132.7 (CH=), 147.9 (C-6), 148.5 (C-7), 171.2 (CO) ppm. HRMS calcd. for $\mathrm{C}_{14} \mathrm{H}_{17} \mathrm{NO}_{3}[\mathrm{M}+\mathrm{H}]^{+}$: 248.1288; found 248.1278.

(1S)-1-[2-(1,3-Dioxan-2-yl)ethyl]-6,7-dimethoxy-3-oxo-1,2,3,4tetrahydroisoquinoline (6i): Operating as described in the general procedure, from $5 \mathbf{i}(114 \mathrm{mg}, 0.26 \mathrm{mmol})$ in THF $(2 \mathrm{~mL})$ and $\mathrm{NH}_{3}$ $(30 \mathrm{~mL})$ a residue was obtained. Flash chromatography $(9: 1$ EtOAc-EtOH) afforded $\mathbf{6 i}(78.5 \mathrm{mg}, 92 \%)$ as a white solid. $[\alpha]^{22}{ }_{\mathrm{D}}$ $=+2.3\left(c=0.53, \mathrm{CHCl}_{3}\right)$. IR $(\mathrm{KBr}): \mathrm{v}=1674,3217 \mathrm{~cm}^{-1} .{ }^{1} \mathrm{H}$ NMR $\left(400 \mathrm{MHz}, \mathrm{CDCl}_{3}, 25^{\circ} \mathrm{C}\right): \delta=1.33(\mathrm{dm}, \mathrm{J}=13.2 \mathrm{~Hz}, 2 \mathrm{H}, \mathrm{H}-$ 2'), 1.61-1.84 (m, 2H, H-1'), 1.87-1.94 (m, $1 \mathrm{H}, \mathrm{CH}_{2} \mathrm{CH}_{2} \mathrm{O}$ ), 1.91$2.11\left(\mathrm{~m}, 1 \mathrm{H}, \mathrm{CH}_{2} \mathrm{CH}_{2} \mathrm{O}\right), 3.56$ (d, $\left.J=19.5 \mathrm{~Hz}, 1 \mathrm{H}, \mathrm{H}-4\right), 3.60$ (d, $J$ $=19.5 \mathrm{~Hz}, 1 \mathrm{H}, \mathrm{H}-4), 3.74\left(\mathrm{td}, J=11.6,0.8 \mathrm{~Hz}, 2 \mathrm{H}, \mathrm{CH}_{2} \mathrm{CH}_{2} \mathrm{O}\right)$, 3.86 (s, $3 \mathrm{H}, \mathrm{OCH}_{3}$ ), 3.87 (s, $3 \mathrm{H}, \mathrm{OCH}_{3}$ ), 4.09 (ddd, $J=11.6,4.8$, $1.2 \mathrm{~Hz}, 2 \mathrm{H}, \mathrm{CH}_{2} \mathrm{CH}_{2} \mathrm{O}$ ), 4.48-4.52 (br m, $\left.1 \mathrm{H}, \mathrm{H}-1\right), 4.56$ (t, $J=4.8$ $\mathrm{Hz}, 1 \mathrm{H}, \mathrm{CHO}_{2}$ ), 6.59 (s, 1H, H-8), 6.65 (s, 1H, H-5), 7.18 (s.a., $1 \mathrm{H}$, $\mathrm{NH})$ ppm. ${ }^{13} \mathrm{C}$ NMR $\left(100.6 \mathrm{MHz}, \mathrm{CDCl}_{3}, 25^{\circ} \mathrm{C}\right): \delta=25.7$ $\left(\mathrm{CH}_{2} \mathrm{CH}_{2} \mathrm{O}\right), 30.5$ (C-1'), 33.0 (C-2'), 35.3 (C-4), 55.6 (C-1), 55.9 $\left(\mathrm{OCH}_{3}\right), 56.0\left(\mathrm{OCH}_{3}\right), 66.8\left(\mathrm{CH}_{2} \mathrm{O}\right), 101.6\left(\mathrm{CHO}_{2}\right), 108.8(\mathrm{C}-8)$, 110.4 (C-5), 123.1 (C-4a), 126.6 (C-8a), 147.8 (C-6), 148.3 (C-7), 171.4 (CO) ppm. HRMS calcd. for $\mathrm{C}_{17} \mathrm{H}_{23} \mathrm{NO}_{5}[\mathrm{M}+\mathrm{H}]^{+}$: 322.1655 ; found 322.1650 .

2-Benzyl-4,5-dimethoxyphenylacetamide (8): Operating as described in the general procedure, from $\mathbf{5 c}(100 \mathrm{mg}, 1.17 \mathrm{mmol})$ in THF $(2 \mathrm{~mL})$ and $\mathrm{NH}_{3}(30 \mathrm{~mL})$ a residue was obtained. Flash chromatography (EtOAc) afforded 8 (20 mg, 28\%). IR (KBr): $v=1629,2933 \mathrm{~cm}^{-1} .{ }^{1} \mathrm{H}$ NMR $\left(400 \mathrm{MHz}, \mathrm{CDCl}_{3}, 25^{\circ} \mathrm{C}\right): \delta=3.44$ (s, $\left.2 \mathrm{H}, \mathrm{CH}_{2} \mathrm{CO}\right), 3.81$ (s, $\left.3 \mathrm{H}, \mathrm{OCH}_{3}\right), 3.85\left(\mathrm{~s}, 3 \mathrm{H}, \mathrm{OCH}_{3}\right), 3.93$ (s, $\left.2 \mathrm{H}, \mathrm{CH}_{2} \mathrm{Ar}\right), 5.20\left(\mathrm{~s}, 1 \mathrm{H}, \mathrm{NH}_{2}\right), 5.90\left(\mathrm{~s}, 1 \mathrm{H}, \mathrm{NH}_{2}\right), 6.70(\mathrm{~s}, 1 \mathrm{H}, \mathrm{H}-$ 3), $6.75(\mathrm{~s}, 1 \mathrm{H}, \mathrm{H}-6), 7.10-7.25(\mathrm{~m}, 5 \mathrm{H}, \mathrm{ArH}) \mathrm{ppm} .{ }^{13} \mathrm{C} \mathrm{NMR}$ $\left(100.6 \mathrm{MHz}, \mathrm{CDCl}_{3}, 25^{\circ} \mathrm{C}\right): \delta=38.8\left(\mathrm{CH}_{2} \mathrm{Ar}\right), 40.2\left(\mathrm{CH}_{2} \mathrm{CO}\right), 55.9$ $\left(\mathrm{OCH}_{3}\right), 56.0\left(\mathrm{OCH}_{3}\right), 113.8(\mathrm{C}-3), 114.2(\mathrm{C}-6), 125.5(\mathrm{C}-1), 126.3$ (C-p), 128.5 (C-m), 128.6 (C-o), 131.6 (C-2), 140.3 (C-i), 147.8 (C-4), 148.3 (C-5), 173.9 (CO) ppm. HRMS calcd. for $\mathrm{C}_{17} \mathrm{H}_{19} \mathrm{NO}_{3}$ $[\mathrm{M}+\mathrm{H}]^{+}:$286.1443; found 286.1437.

General Procedure for the $\mathrm{NaBH}_{4}-\mathrm{I}_{2}$ Reduction Reactions: A solution of iodine (1 equiv) in THF was slowly added to a cooled $\left(0{ }^{\circ} \mathrm{C}\right)$ suspension of $\mathrm{NaBH}_{4}$ (2.5 equiv) in anhydrous THF, and the mixture was stirred at this temperature for $30 \mathrm{~min}$. Then, a solution of lactam 6 ( 1 equiv) in THF was added to the solution $\left(0^{\circ} \mathrm{C}\right)$. The resulting mixture was refluxed for $16 \mathrm{~h}$ and cooled to $0^{\circ} \mathrm{C}$. $\mathrm{MeOH}$ $(4 \mathrm{~mL})$ was slowy added, and the stirring was continued at room temperature for $30 \mathrm{~min}$. The solvent was evaporated, and the resulting solid was digested with $2 \mathrm{~N} \mathrm{NaOH}$ (30 min). The resulting 
suspension was extracted with $\mathrm{CH}_{2} \mathrm{Cl}_{2}$, the combined organic extracts were dried and concentrated, and the residue was chromatographed.

(-)-Salsolidine (7a): Following the above general procedure, from lactam 6a (100 mg, $0.45 \mathrm{mmol})$ in THF $(5 \mathrm{~mL}), \mathrm{NaBH}_{4}(42.6 \mathrm{mg}$, $1.13 \mathrm{mmol})$ in THF $(5 \mathrm{~mL})$, and $\mathrm{I}_{2}(114 \mathrm{mg}, 0.45 \mathrm{mmol})$ in THF (4 $\mathrm{mL})$, tetrahydroisoquinoline $7 \mathbf{a}(65 \mathrm{mg}, 70 \%)$ was obtained as an oil after flash chromatography $\left(9: 1\right.$ EtOAc-EtOH). $[\alpha]^{22}{ }_{\mathrm{D}}=-58.5$ $\left(c=0.50\right.$, EtOH) $\left\{\right.$ lit.. $\left.{ }^{[1 b]}[\alpha]^{24}-62.5(c 0.1, \mathrm{EtOH})\right\}$. IR (KBr): $v=3217 \mathrm{~cm}^{-1} .{ }^{1} \mathrm{H}$ NMR $\left(300 \mathrm{MHz}, \mathrm{CDCl}_{3}, 25^{\circ} \mathrm{C}\right): \delta=1.43(\mathrm{~d}, J=$ $6.5 \mathrm{~Hz}, 3 \mathrm{H}, \mathrm{CH}_{3}$ ), 1.78 (br s, $1 \mathrm{H}, \mathrm{NH}$ ), 2.60-2.68 (dt, $J=16.0,4.8$ $\mathrm{Hz}, 1 \mathrm{H}, \mathrm{H}-4), 2.74-2.84$ (ddd, $J=16.0,8.4,5.4 \mathrm{~Hz}, 1 \mathrm{H}, \mathrm{H}-4), 2.99$ (ddd, $J=13.0,8.4,4.8 \mathrm{~Hz}, 1 \mathrm{H}, \mathrm{H}-3$ ), 3.24 (ddd, $\mathrm{J}=13.0,4.8 \mathrm{~Hz}$, $1 \mathrm{H}, \mathrm{H}-3), 3.87$ (s, 6H, 2OCH 3 ), 4.04 (q, $J=6.5 \mathrm{~Hz}, 1 \mathrm{H}, \mathrm{H}-1), 6.57$ (s, $1 \mathrm{H}, \mathrm{H}-8), 6.62(\mathrm{~s}, 1 \mathrm{H}, \mathrm{H}-5) \mathrm{ppm} .{ }^{13} \mathrm{C} \mathrm{NMR}\left(100.6 \mathrm{MHz}, \mathrm{CDCl}_{3}\right.$, $\left.25^{\circ} \mathrm{C}\right): \delta=22.8\left(\mathrm{CH}_{3}\right), 29.5(\mathrm{C}-4), 41.8(\mathrm{C}-3), 51.2(\mathrm{C}-1), 55.8$ $\left(\mathrm{OCH}_{3}\right), 55.9\left(\mathrm{OCH}_{3}\right), 109.0(\mathrm{C}-8), 111.7(\mathrm{C}-5), 126.8(\mathrm{C}-4 \mathrm{a})$, 132.5 (C-8a), 147.2 (C-7), 147.3 (C-6) ppm.

\section{(1S)-1-Ethyl-6,7-dimethoxy-1,2,3,4-tetrahydroisoquinoline}

(7b): Following the above general procedure, from lactam $\mathbf{6 b}$ (91 $\mathrm{mg}, 0.39 \mathrm{mmol})$ in THF $(10 \mathrm{~mL}), \mathrm{NaBH}_{4}(36 \mathrm{mg}, 1.1 \mathrm{mmol})$ in THF $(4 \mathrm{~mL})$, and $\mathrm{I}_{2}(99 \mathrm{mg}, 0.39 \mathrm{mmol})$ in THF $(4 \mathrm{~mL})$, tetrahydroisoquinoline $7 \mathbf{b}(52 \mathrm{mg}, 60 \%)$ was obtained as an oil after flash chromatography using a cartridge containing amine functionalized silica $\left(7: 3\right.$ hexane-EtOAc to EtOAc). $[\alpha]^{22}{ }_{\mathrm{D}}=-$ $47.4\left(c=0.3, \mathrm{CH}_{2} \mathrm{Cl}_{2}\right)\left\{\right.$ lit.: ${ }^{[25]}[\alpha]_{\mathrm{D}}^{22}-51.9\left(c\right.$ 2.1, $\left.\left.\mathrm{CH}_{2} \mathrm{Cl}_{2}\right)\right\}$. IR $(\mathrm{KBr}): v=2930 \mathrm{~cm}^{-1} .{ }^{1} \mathrm{H}$ NMR $\left(400 \mathrm{MHz}, \mathrm{CDCl}_{3}, 25^{\circ} \mathrm{C}\right): \delta=1.02$ (t, $\left.J=7.6 \mathrm{~Hz}, 3 \mathrm{H}, \mathrm{CH}_{3}\right), 1.67-1.78\left(\mathrm{~m}, 1 \mathrm{H}, \mathrm{CH}_{2}\right), 1.90$ (dddd, $J=$ 14.4, 7.2, 7.2, $\left.3.2 \mathrm{~Hz}, 1 \mathrm{H}, \mathrm{CH}_{2}\right), 2.40(\mathrm{~s}, 1 \mathrm{H}, \mathrm{NH}), 2.67(\mathrm{dt}, J=$ 16.2, 5.2 Hz, 1H, H-3), 2.77 (dt, $J=16.2,6.0 \mathrm{~Hz}, 1 \mathrm{H}, \mathrm{H}-3), 2.98$ (ddd, $J=12.4,7.6,4.8 \mathrm{~Hz}, 1 \mathrm{H}, \mathrm{H}-4), 3.24$ (dt, $J=12.4,5.2 \mathrm{~Hz}, 1 \mathrm{H}$, $\mathrm{H}-4), 3.85$ (s, $\left.6 \mathrm{H}, 2 \mathrm{OCH}_{3}\right), 3.85$ (m, 1H, H-1), 6.57 (s, 1H, H-8), 6.62 (s, $1 \mathrm{H}, \mathrm{H}-5) \mathrm{ppm} .{ }^{13} \mathrm{C} \mathrm{NMR}\left(100.6 \mathrm{MHz}, \mathrm{CDCl}_{3}, 25^{\circ} \mathrm{C}\right): \delta=$ $10.9\left(\mathrm{CH}_{3}\right), 29.0\left(\mathrm{CH}_{2}\right), 29.4(\mathrm{C}-4), 41.1(\mathrm{C}-3), 55.8\left(\mathrm{OCH}_{3}\right), 56.0$ $\left(\mathrm{OCH}_{3}\right), 56.7$ (C-1), 109.2 (C-8), 111.7 (C-5), 127.1 (C-4a), 131.0 (C-8a), 147.2 (C-7), 147.3 (C-6) ppm. HRMS calcd. for $\mathrm{C}_{13} \mathrm{H}_{19} \mathrm{NO}_{2}$ $[\mathrm{M}+\mathrm{H}]^{+}:$222.1494; found 222.1488 .

(-)-Norcryptostyline II (7d): Following the above general procedure, from lactam $\mathbf{6 d}(90 \mathrm{mg}, 0.26 \mathrm{mmol})$ in THF $(3 \mathrm{~mL})$, $\mathrm{NaBH}_{4}(24.8 \mathrm{mg}, 0.66 \mathrm{mmol})$ in THF $(4 \mathrm{~mL})$, and $\mathrm{I}_{2}(66.5 \mathrm{mg}, 0.26$ $\mathrm{mmol})$ in THF (3 mL), tetrahydroisoquinoline $7 \mathbf{d}(58 \mathrm{mg}, 69 \%)$ was obtained as a yelow oil after flash chromatography using a cartridge containing amine functionalized silica (1:1 hexaneEtOAc). $[\alpha]^{22}{ }_{\mathrm{D}}=-33.8\left(c=0.36, \mathrm{CHCl}_{3}\right)\left\{1 \mathrm{it} . .^{[5 \mathrm{a}]}[\alpha]_{\mathrm{D}}^{18}-37(\mathrm{c}\right.$ $\left.\left.0.26, \mathrm{CHCl}_{3}\right)\right\}$. IR (KBr): $v=2923 \mathrm{~cm}^{-1}$. ${ }^{1} \mathrm{H}$ NMR $(400 \mathrm{MHz}$, $\left.\mathrm{CDCl}_{3}, 25^{\circ} \mathrm{C}\right): \delta=2.75(\mathrm{dt}, J=15.2,4.4 \mathrm{~Hz}, 1 \mathrm{H}, \mathrm{H}-4), 2.96(\mathrm{ddd}$, $J=15.2,4.8,4.8 \mathrm{~Hz}, 1 \mathrm{H}, \mathrm{H}-4), 3.06$ (ddd, $J=13.2,8.8,4.8 \mathrm{~Hz}$, $1 \mathrm{H}, \mathrm{H}-3$ ), 3.24 (ddd, $J=13.2,4.8 \mathrm{~Hz}, 1 \mathrm{H}, \mathrm{H}-3), 3.65$ (s, $3 \mathrm{H}$, $\left.\mathrm{OCH}_{3}\right), 3.83\left(\mathrm{~s}, 3 \mathrm{H}, \mathrm{OCH}_{3}\right), 3.87\left(\mathrm{~s}, 6 \mathrm{H}, \mathrm{OCH}_{3}\right), 5.00(\mathrm{~s}, 1 \mathrm{H}, \mathrm{H}-1)$, 6.27 (s, 1H, H-8), 6.62 (s, 1H, H-5), 6.79 (s, 1H, H-2'), 6.80 (s, 1H, $\mathrm{H}-6$ '), 6.82 (s, 1H, H-5') ppm. ${ }^{13} \mathrm{C}$ NMR (75.4 $\mathrm{MHz}, \mathrm{CDCl}_{3}$, $\left.25^{\circ} \mathrm{C}\right): \delta=29.1(\mathrm{C}-4), 42.1(\mathrm{C}-3), 55.8\left(\mathrm{OCH}_{3}\right), 55.9\left(\mathrm{OCH}_{3}\right), 56.0$ $\left(\mathrm{OCH}_{3}\right), 61.3(\mathrm{C}-1), 110.7$ (C-2'), 110.9 (C-5'), $111.4(\mathrm{C}-8), 111.8$ (C-5), 121.3 (C-6'), 127.4 (C-4a), 129.8 (C-8a), 136.9 (C-i), 147.0 (C-6), 147.7 (C-4'), 148.4 (C-7), 149.0 (C-3') ppm. HRMS calcd. for $\mathrm{C}_{19} \mathrm{H}_{23} \mathrm{NO}_{4}[\mathrm{M}+\mathrm{H}]^{+}: 330.1705$; found 330.1691.
(-)-Norcryptostyline III (7e): Following the above general procedure, from lactam $6 \mathrm{e}(92 \mathrm{mg}, 0.25 \mathrm{mmol})$ in THF $(3 \mathrm{~mL})$, $\mathrm{NaBH}_{4}(23.3 \mathrm{mg}, 0.62 \mathrm{mmol})$ in THF $(4 \mathrm{~mL})$, and $\mathrm{I}_{2}(70.8 \mathrm{mg}, 0.25$ $\mathrm{mmol})$ in THF $(3 \mathrm{~mL})$, tetrahydroisoquinoline 7 e $(52 \mathrm{mg}, 58 \%)$ was obtained as a yelow oil after flash chromatography using a cartridge containing amine functionalized silica (7:3 $\left.\mathrm{Et}_{2} \mathrm{O}-\mathrm{EtOAc}\right)$. $[\alpha]_{D}^{22}=-45.7\left(c=0.11, \mathrm{CHCl}_{3}\right)\left\{\right.$ lit.. $\left.{ }^{[16 \mathrm{a}]}[\alpha]_{\mathrm{D}}-37.0\left(\mathrm{CHCl}_{3}\right)\right\}$. IR (KBr): $v=2919 \mathrm{~cm}^{-1} .{ }^{1} \mathrm{H}$ NMR (300 MHz, $\left.\mathrm{CDCl}_{3}, 25^{\circ} \mathrm{C}\right): \delta=$ 2.74 (dt, $J=15.6,4.5,3.9 \mathrm{~Hz}, 1 \mathrm{H}, \mathrm{H}-4), 2.90-3.00$ (m, 1H, H-4), 3.07 (ddd, $J=11.7,7.8,3.9 \mathrm{~Hz}, 1 \mathrm{H}, \mathrm{H}-3), 3.26$ (dt, $J=11.7,5.1$, $4.5 \mathrm{~Hz}, 1 \mathrm{H}, \mathrm{H}-3), 3.68\left(\mathrm{~s}, 3 \mathrm{H}, \mathrm{OCH}_{3}\right), 3.81\left(\mathrm{~s}, 6 \mathrm{H}, 2 \mathrm{CH}_{3} \mathrm{O}\right), 3.85(\mathrm{~s}$, $\left.3 \mathrm{H}, \mathrm{OCH}_{3}\right), 3.88\left(\mathrm{~s}, 3 \mathrm{H}, \mathrm{OCH}_{3}\right), 5.01(\mathrm{~s}, 1 \mathrm{H}, \mathrm{H}-1), 6.26(\mathrm{~s}, 1 \mathrm{H}, \mathrm{H}-$ 8), 6.47 (s, 2H, H-2', H-6'), 6.63 (s, 1H, H-5) ppm. ${ }^{13} \mathrm{C}$ NMR (75.4 $\left.\mathrm{MHz}, \mathrm{CDCl}_{3}, 25^{\circ} \mathrm{C}\right): \delta=27.9(\mathrm{C}-4), 41.5(\mathrm{C}-3), 55.8\left(\mathrm{OCH}_{3}\right), 56.0$ $\left(\mathrm{OCH}_{3}\right), 56.2\left(\mathrm{OCH}_{3}\right), 60.8\left(\mathrm{OCH}_{3}\right), 61.2(\mathrm{C}-1), 106.4\left(\mathrm{C}-2{ }^{\prime}, \mathrm{C}-6{ }^{\prime}\right)$, 110.8 (C-8), 111.3 (C-5), 126.7 (C-4a), 129.8 (C-8a), 137.7 (C-1'), 147.4 (C-6), 148.2 (C-7), 153.2 (C-3', C-4', C-5') ppm. HRMS calcd. for $\mathrm{C}_{20} \mathrm{H}_{25} \mathrm{NO}_{5}[\mathrm{M}+\mathrm{H}]^{+}: 360.1809$; found 360.1809 .

(-)-O,O-Dimethylcoclaurine (7f): Following the above general procedure, from lactam 6 f $(200 \mathrm{mg}, 0.6 \mathrm{mmol})$ in THF $(3 \mathrm{~mL})$, $\mathrm{NaBH}_{4}(58 \mathrm{mg}, 1.5 \mathrm{mmol})$ in THF $(4 \mathrm{~mL})$, and $\mathrm{I}_{2}(152 \mathrm{mg}, 0.8$ $\mathrm{mmol})$ in THF (3 mL), tetrahydroisoquinoline $7 \mathbf{f}(111 \mathrm{mg}, 59 \%)$ was obtained as a yelow oil after flash chromatography using a cartridge containing amine functionalized silica (8:2 to $1: 1$ hexaneEtOAc). $[\alpha]^{22}{ }_{\mathrm{D}}=-11.8\left(c=0.5, \mathrm{CHCl}_{3}\right)\left\{\right.$ lit.. ${ }^{[18 c]}[\alpha]^{22}{ }_{\mathrm{D}}-19.9(c 1$, $\left.\left.\mathrm{CHCl}_{3}\right)\right\}$. IR (KBr): $\mathrm{v}=2932 \mathrm{~cm}^{-1}$. ${ }^{1} \mathrm{H}$ NMR $\left(400 \mathrm{MHz}, \mathrm{CDCl}_{3}\right.$, $\left.25^{\circ} \mathrm{C}\right): \delta=2.64-2.79(\mathrm{~m}, 1 \mathrm{H}, \mathrm{H}-3), 2.73(\mathrm{ddd}, J=11.6,6.0,6.0 \mathrm{~Hz}$, $1 \mathrm{H}, \mathrm{H}-4), 2.86$ (dd, $J=14.0,9.5 \mathrm{~Hz}, 1 \mathrm{H}, \mathrm{CH}_{2} \mathrm{Ar}$ ), 2.91 (ddd, $J=$ 12.0, 6.0, $5.6 \mathrm{~Hz}, 1 \mathrm{H}, \mathrm{H}-3$ ), 3.15 (dd, $J=14.0,4.5 \mathrm{~Hz}, 1 \mathrm{H}, \mathrm{CH}_{2} \mathrm{Ar}$ ), 3.20 (ddd, $J=11.6,11.6,5.6 \mathrm{~Hz}, 1 \mathrm{H}, \mathrm{H}-4), 3.80$ (s, $1 \mathrm{H}, \mathrm{OCH}_{3}$ ), $3.82\left(\mathrm{~s}, 1 \mathrm{H}, \mathrm{OCH}_{3}\right), 3.86\left(\mathrm{~s}, 1 \mathrm{H}, \mathrm{OCH}_{3}\right), 4.11(\mathrm{dd}, J=9.5,4.5 \mathrm{~Hz}$, $1 \mathrm{H}, \mathrm{H}-1), 6.59$ (s, 1H, H-8), 6.63 (s, 1H, H-5), 6.86 (d, $J=8.5 \mathrm{~Hz}$, $2 \mathrm{H}, \mathrm{H}-3$ ') 7.16 (d, $J=8.5 \mathrm{~Hz}, 2 \mathrm{H}, \mathrm{H}-5$ ') ppm. ${ }^{13} \mathrm{C}$ NMR $(100.6$ $\left.\mathrm{MHz}, \mathrm{CDCl}_{3}, 25^{\circ} \mathrm{C}\right): \delta=29.5(\mathrm{C}-4), 40.7\left(\mathrm{CH}_{2} \mathrm{Ar}\right), 41.5(\mathrm{C}-3)$, $55.3\left(\mathrm{OCH}_{3}\right), 55.8\left(\mathrm{OCH}_{3}\right), 55.9\left(\mathrm{OCH}_{3}\right), 56.9(\mathrm{C}-1), 109.4(\mathrm{C}-8)$, 111.8 (C-5), 114.0 (C-3'), 127.3 (C-4a), 130.3 (C-2'), 130.5 (C-1'), 131.0 (C-8a), 147.0 (C-7), 147.4 (C-6), 158.3 (C-4') ppm. HRMS calcd. for $\mathrm{C}_{19} \mathrm{H}_{23} \mathrm{NO}_{3}[\mathrm{M}+\mathrm{H}]^{+}$: 314.1756 ; found 314.1743 .

\section{(1S)-6,7-Dimethoxy-1-phenylethyl-1,2,3,4-}

tetrahydroisoquinoline (7g): Following the above general procedure, from lactam $6 \mathbf{g}(250 \mathrm{mg}, 0.8 \mathrm{mmol})$ in THF $(3 \mathrm{~mL})$, $\mathrm{NaBH}_{4}(76 \mathrm{mg}, 2.0 \mathrm{mmol})$ in THF $(4 \mathrm{~mL})$, and $\mathrm{I}_{2}(203 \mathrm{mg}, 0.8$ $\mathrm{mmol})$ in THF (3 mL), tetrahydroisoquinoline $7 \mathrm{~g}(183 \mathrm{mg}, 77 \%)$ was obtained as a colourless oil after flash chromatography $\left(\mathrm{SiO}_{2}\right.$ previously washed with 8:2 $\mathrm{Et}_{3} \mathrm{~N}-\mathrm{EtOAc} ; 8: 2$ to $1: 1 \mathrm{Et}_{2} \mathrm{O}-\mathrm{EtOAc}$ as eluent $) .[\alpha]^{22}{ }_{D}=-23.4\left(c=0.25, \mathrm{CHCl}_{3}\right)$. IR $(\mathrm{KBr}): v=2955$ $\mathrm{cm}^{-1}$. ${ }^{1} \mathrm{H}$ NMR $\left(400 \mathrm{MHz}, \mathrm{CDCl}_{3}, 25^{\circ} \mathrm{C}\right): \delta=2.04-2.17(\mathrm{~m}, 2 \mathrm{H}$, $\mathrm{CH}_{2}$ ), 2.66-2.89 (m, 4H, $\left.\mathrm{CH}_{2} \mathrm{Ar}, \mathrm{H}-4\right), 3.02$ (ddd, $J=12.0,7.2,5.6$ $\mathrm{Hz}, 1 \mathrm{H}, \mathrm{H}-3$ ), 3.27 (ddd, $J=12.0,5.6,5.6 \mathrm{~Hz}, 1 \mathrm{H}, \mathrm{H}-3$ ), 3.82 (s, $\left.3 \mathrm{H}, \mathrm{OCH}_{3}\right), 3.85\left(\mathrm{~s}, 3 \mathrm{H}, \mathrm{OCH}_{3}\right), 4.00(\mathrm{dd}, J=8.0,2.8 \mathrm{~Hz}, 1 \mathrm{H}, \mathrm{H}-$ 1), 6.57 (s, 2H, H-5, H-8), 7.17-7.31 (m, 5H, ArH) ppm. ${ }^{13} \mathrm{C}$ NMR $\left(75.4 \mathrm{MHz}, \mathrm{CDCl}_{3}, 25^{\circ} \mathrm{C}\right): \delta=29.1\left(\mathrm{CH}_{2} \mathrm{Ar}\right), 32.4(\mathrm{C}-4), 38.1$ $\left(\mathrm{CH}_{2}\right), 40.9(\mathrm{C}-3), 55.1(\mathrm{C}-1), 55.8\left(\mathrm{OCH}_{3}\right), 56.0\left(\mathrm{OCH}_{3}\right), 109.2$ (C-8), 111.8 (C-5), 125.9 (C-4'), 127.0 (C-4a), 128.4 (C-2', C-3'), 128.5 (C-8a), 142.2 (C-4'), 147.3 (C-6), 147.4 (C-7) ppm. HRMS calcd. for $\mathrm{C}_{19} \mathrm{H}_{23} \mathrm{NO}_{2}[\mathrm{M}+\mathrm{H}]^{+}$: 298.1807; found 298.1802. 
(1S)-1-[2-(1,3-Dioxan-2-yl)ethyl]-6,7-dimethoxy-1,2,3,4tetrahydroisoquinoline (9): $\mathrm{LiAlH}_{4}(96 \mathrm{mg}, 2.54 \mathrm{mmol})$ was slowly added to a suspension of $\mathrm{AlCl}_{3}(113 \mathrm{mg}, 0.85 \mathrm{mmol})$ in THF $(6 \mathrm{~mL})$ at $-78^{\circ} \mathrm{C}$ and the mixture was stirred for $2 \mathrm{~h}$. Then, a solution of tetrahydroisoquinolone $\mathbf{5 i}(170 \mathrm{mg}, 0.39 \mathrm{mmol})$ in anhydrous THF $(6 \mathrm{~mL})$ was slowly added. The stirring was continued at $-78{ }^{\circ} \mathrm{C}$ for $20 \mathrm{~h}$, and the reaction was quenched with water. The aqueous layer was extracted with $\mathrm{CH}_{2} \mathrm{Cl}_{2}$, and the combined organic extracts were dried and concentrated to give a residue, which was chromatographed $\left(\mathrm{Et}_{2} \mathrm{O}\right.$ to $\left.1: 1 \mathrm{Et}_{2} \mathrm{O}-\mathrm{EtOAc}\right)$ to afford tetrahydroisoquinoline 9 (144 mg, 87\%). [ $\alpha]_{D}^{22}=-37.1 \quad(c$ $\left.=1.03, \mathrm{CHCl}_{3}\right)$. IR (KBr): $v=3399 \mathrm{~cm}^{-1} .{ }^{1} \mathrm{H}$ NMR $(400 \mathrm{MHz}$, $\left.\mathrm{CDCl}_{3}, 25^{\circ} \mathrm{C}\right): \delta=1.26-1.31\left(\mathrm{~m}, 1 \mathrm{H}, \mathrm{ClCH}_{2}\right), 1.49-1.52(\mathrm{~m}, 1 \mathrm{H}$, $\left.\mathrm{OCH}_{2} \mathrm{CH}_{2}\right), 1.53-1.62\left(\mathrm{~m}, 1 \mathrm{H}, \mathrm{CH}_{2} \mathrm{CHO}_{2}\right), 1.76-1.82(\mathrm{~m}, 1 \mathrm{H}$, $\left.\mathrm{CH}_{2} \mathrm{CHO}_{2}\right), 1.83-1.93\left(\mathrm{~m}, 1 \mathrm{H}, \mathrm{OCH}_{2} \mathrm{CH}_{2}\right), 1.97-2.09(\mathrm{~m}, 1 \mathrm{H}$, $\left.\mathrm{C}_{1 \mathrm{CH}_{2}}\right), 2.27(\mathrm{~s}, 1 \mathrm{H}, \mathrm{OH}), 2.43(\mathrm{dd}, J=17.0,5.0 \mathrm{~Hz}, 1 \mathrm{H}, \mathrm{H}-4)$, 2.97 (ddd, J = 17.0, 12.0, $6.0 \mathrm{~Hz}, 1 \mathrm{H}, \mathrm{H}-4), 3.20$ (dd, $J=13.5,6.0$ $\mathrm{Hz}, 1 \mathrm{H}, \mathrm{H}-3), 3.31$ (dd, $J=13.5,5.0 \mathrm{~Hz}, 1 \mathrm{H}, \mathrm{H}-3), 3.37$ (dd, $J=$ 9.6, 4.4 Hz, $1 \mathrm{H}, \mathrm{H}-1), 3.64-3.67$ (m, $\left.2 \mathrm{H}, 2 \mathrm{OCH}_{2} \mathrm{CH}_{2}\right), 3.68-3.73$ (m, $1 \mathrm{H}, \mathrm{CHAr}), 3.75\left(\mathrm{~s}, 3 \mathrm{H}, \mathrm{OCH}_{3}\right), 3.83\left(\mathrm{~s}, 3 \mathrm{H}, \mathrm{OCH}_{3}\right), 3.81-3.86$ (m, $2 \mathrm{H}, 2 \mathrm{OCH}_{2} \mathrm{CH}_{2}$ ), $3.95\left(\mathrm{dd}, J=10.8,6.0 \mathrm{~Hz}, 1 \mathrm{H}, \mathrm{CH}_{2} \mathrm{OH}\right), 4.05$ (dd, $J=10.8,4.8 \mathrm{~Hz}, 1 \mathrm{H}, \mathrm{CH}_{2} \mathrm{OH}$ ), 4.40 (t, $J=4.8 \mathrm{~Hz}, 1 \mathrm{H}, \mathrm{CHO}_{2}$ ), 6.28 (s, 1H, H-8), 6.55 (s, 1H, H-5), 7.28-7.29 (m, 5H, ArH) ppm. ${ }^{13} \mathrm{C}$ NMR $\left(75.4 \mathrm{MHz}, \mathrm{CDCl}_{3}, 25^{\circ} \mathrm{C}\right): \delta=22.5\left(\mathrm{CH}_{2} \mathrm{CH}_{2} \mathrm{O}\right), 25.6$ (C-4), 30.7 (C-1'), 32.1 (C-2'), $39.0(\mathrm{C}-3), 55.6\left(\mathrm{OCH}_{3}\right), 55.7$ $\left(\mathrm{OCH}_{3}\right), 57.6(\mathrm{C}-1), 64.0\left(\mathrm{CH}_{2} \mathrm{OH}\right), 64.5(\mathrm{CHAr}), 66.6\left(\mathrm{OCH}_{2} \mathrm{CH}_{2}\right)$, $102.1\left(\mathrm{CHO}_{2}\right), 110.5(\mathrm{C}-8), 111.3(\mathrm{C}-5), 125.4(\mathrm{C}-4 \mathrm{a}), 127.4(\mathrm{C}-p)$, 128.2 (C-o), 128.6 (C-m), 130.3 (C-8a), 140.9 (C-i), 146.9 (C-6), 147.1 (C-7) ppm. HRMS calcd. for $\mathrm{C}_{25} \mathrm{H}_{33} \mathrm{NO}_{5}[\mathrm{M}+\mathrm{H}]^{+}$: 428.2437; found 428.2424 .

(S)-(-)-Crispine A: A solution of tetrahydroisoquinoline 9 (110 $\mathrm{mg}, 0.26 \mathrm{mmol})$ in $\mathrm{EtOH}(12 \mathrm{~mL})$ and $1.0 \mathrm{M}$ aqueous $\mathrm{HCl}(0.5$ $\mathrm{mL})$ containing $10 \% \mathrm{Pd}-\mathrm{C}(15 \mathrm{mg})$ was hydrogenated with vigorous stirring at room temperature and atmospheric pressure for 3 days. The catalyst was removed by filtration, the solvent was concentrated under vacuum, and the resulting oil was chromatographed using a cartridge containing amine functionalized silica (1:1 hexane-EtOAc) to give crispine A (45 mg, 74\%). $[\alpha]^{22}{ }_{D}$ $=-100.1\left(c=0.32, \mathrm{CHCl}_{3}\right)\left\{\right.$ lit. $\left.^{\left[{ }^{[26]}\right.}[\alpha]^{22}{ }_{\mathrm{D}}-96\left(c 0.25, \mathrm{CHCl}_{3}\right)\right\}$. IR (KBr): $v=2922 \mathrm{~cm}^{-1} .{ }^{1} \mathrm{H}$ NMR $\left(400 \mathrm{MHz}, \mathrm{CDCl}_{3}, 25^{\circ} \mathrm{C}\right): \delta=$ 1.69-1.78 (m, 1H, H-1), 1.82-1.99 (m, 2H, H-2), 2.30-2.38 (m, $1 \mathrm{H}$, $\mathrm{H}-1$ ), 2.63 (ddd, $J=17.0,8.0,8.0 \mathrm{~Hz}, 1 \mathrm{H}, \mathrm{H}-6), 2.69$ (m, 1H, H-5), $2.74(\mathrm{~m}, 1 \mathrm{H}, \mathrm{H}-3), 3.00$ (ddd, $J=12.4,9.6,5.6 \mathrm{~Hz}, 1 \mathrm{H}, \mathrm{H}-3), 3.07$ (ddd, $J=17.0,8.0,4.0 \mathrm{~Hz}, 1 \mathrm{H}, \mathrm{H}-6$ ), 3.18 (dddd, $J=17.2,11.2$, 6.4, $2.8 \mathrm{~Hz}, 1 \mathrm{H}, \mathrm{H}-5), 3.49$ (m, 1H, H-10b), 3.85 (s, 6H, $\mathrm{OCH}_{3}$ ), 6.57 (s, 1H, H-10), 6.61 (s, 1H, H-7) ppm. ${ }^{13} \mathrm{C}$ NMR (75.4 MHz, $\left.\mathrm{CDCl}_{3}, 25^{\circ} \mathrm{C}\right): \delta=22.3(\mathrm{C}-2), 27.9(\mathrm{C}-6), 30.6(\mathrm{C}-3), 48.2(\mathrm{C}-3)$, $53.1(\mathrm{C}-5), 55.9\left(\mathrm{OCH}_{3}\right), 56.0\left(\mathrm{OCH}_{3}\right), 62.8(\mathrm{C}-10 \mathrm{~b}), 108.8(\mathrm{C}-10)$, 111.3 (C-7), 126.1 (C-6a), 130.5 (C-10a), 147.3 (C-8), 147.4 (C-9) ppm. HRMS calcd. for $\mathrm{C}_{14} \mathrm{H}_{19} \mathrm{NO}_{2}[\mathrm{M}+\mathrm{H}]^{+}:$234.1494; found 234.1486.

\section{Acknowledgments}

Financial support from the Ministry of Science and Innovation, Spain (Project CTQ2009-07021/BQU), and the AGAUR, Generalitat de Catalunya (Grant 2009-SGR-111) is gratefully acknowledged.

[1] a) The Chemistry and Biology of Isoquinoline Alkaloids, (Ed.: J. D. Phillipson, M. F. Roberts, M. H. Zenk), Springer-Verlag, Berlín,
1985; b) Handbook of Natural Products Data, Vol. 3 (Ed.: Atta-urRahman), Elsevier, Amsterdam, 1994. c) D. Jack, R. Williams, Chem. Rev. 2002, 1669-1730; d) P. M. Dewick in Medicinal Natural Products, Wiley, Chichester, 2002, pp. 315-346; e) K. W. Bentley, Nat. Prod. Rep. 2006, 23, 444-463 and previous reviews in this series.

[2] For reviews, see: a) E. Anakabe, D. Badía, L. Carrillo, E. Reyes, J. L. Vicario, "Chiral $\beta$-Aminoalcohols and Derivatives in the Asymmetric Synthesis of Tetrahydroisoquinolines" in Targets in Heterocyclic Systems, (Eds.: O. A. Attanasi, D. Spinelli), Italian Society of Chemistry, 2002, Vol. 6, pp. 270-311; b) J. L. Vicario, D. Badía, L. Carrillo, J. Etxebarria, Curr. Org. Chem. 2003, 7, 17751792; c) M. Chrzanowska, M. D. Rozwadowska, Chem. Rev. 2004, 104, 3341-3370; d) E. L. Larghi, M. Amongero, A. B. J. Bracca, T. S. Kaufman, Arkivoc 2005 (xii), 98-153; e) Z. Czarnocki, A. Siwicka, J. Szawkato, Curr. Org. Chem. 2005, 2, 301-331.

[3] For reviews on the use of aminoalcohol-derived oxazolopiperidone lactams: a) A. I. Meyers, G. P. Brengel, Chem. Commun. 1997, 1-8; b) Groaning, M. D.; Meyers, A. I. Tetrahedron 2000, 56, 9843-9873. c) C. Escolano, M. Amat, J. Bosch, Chem. Eur. J. 2006, 12, 81988207; d) S. N. Gaskell, L. J. Duffy, S. M. Allin, Nat. Prod. Commun. 2008, 3, 1825-1838. For more recent work, see: e) M. Amat, O. Bassas, N. Llor, M. Cantó, M. Pérez, E. Molins, J. Bosch, Chem. Eur. J. 2006, 12, 7872-7881; f) M. Amat, M. M. M. Santos, O. Bassas, N. Llor, C. Escolano, A. Gómez-Esqué, E. Molins, S. M. Allin, V. McKee, J. Bosch, J. Org. Chem. 2007, 72, 5193-5201; g) M. Amat, R. Griera, R. Fabregat, J. Bosch, Angew. Chem. Int. Ed. 2008, 47, 3348-3351; h) M. Amat, A. Gómez-Esqué, C. Escolano, M. M. M. Santos, E. Molins, J. Bosch, J. Org. Chem. 2009, 74, 1205-1211; i) M. Amat, N. Llor, B. Checa, E. Molins, J. Bosch, J. Org. Chem. 2010, 75, 178-189; j) M. Amat, M. Pérez, S. Proto, T. Gatti, J. Bosch, Chem. Eur. J. 2010, 16, doi: 10.1002/chem.201000421.

[4] T. C. Coombs, M. D. Lee, IV, H. Wong, M. Armstrong, B. Cheng, W. Chen, A. F. Moretto, L. S. Liebeskind J. Org. Chem. 2008, 73, 882-888.

[5] For related approaches from oxazolotetrahydroisoquinolines, see: a) M. Yamato, K. Hashigaki, N. Qais, S. Ishikawa, Tetrahedron 1990, 46, 5909-5920; b) K. Hashigaki, K. Kan, N. Qais, Y. Takeuchi, M. Yamato, Chem. Pharm. Bull. 1991, 39, 1126-1131; c) A.-C. Carbonnelle, V. Gott, G. Roussi, Heterocycles 1993, 36, 1763-1769; d) K. Umetsu, N. Asao, Tetrahedron Lett. 2008, 49, 2722-2725.

[6] J. M. Gardiner, M. R. Bryce, P. A. Bates, M. B. Hursthouse, J. Org. Chem. 1990, 55, 1261-1266.

[7] CCDC-770769 (for 2) contains the supplementary crystallographic data for this paper. These data can be obtained free of charge from The Cambridge Crystallographic Data Centre via www.ccdc.cam.ac.uk/data_request/cif.

[8] a) M. Amat, J. Bosch, J. Hidalgo, M. Cantó, M. Pérez, N. Llor, E. Molins, C. Miravitlles, M. Orozco, J. Luque, J. Org. Chem. 2000, 65, 3074-3084; b) M. Amat, C. Escolano, A. Gómez-Esqué, O. Lozano, N. Llor, R. Griera, E. Molins, J. Bosch, Tetrahedron: Asymmetry 2006, 1581-1588; c) M. Amat, M. Pérez, A. T. Minaglia, D. Passarella, J. Bosch, Tetrahedron: Asymmetry 2008, 19, 2406-2410.

[9] M. Amat, N. Llor, J. Hidalgo, C. Escolano, J. Bosch, J. Org. Chem. 2003, 68, 1919-1928.

[10] The use of $\mathrm{MeMgBr}$ resulted in a lower yield (42\%) of the $\alpha$ amidoalkylation product $\mathbf{5 a}$.

[11] For related $\alpha$-amidoalkylation reactions from oxazolopiperidone lactams, see: M. Amat, C. Escolano, N. Llor, M. Huguet, M. Pérez, J. Bosch, Tetrahedron: Asymmetry 2003, 14, 1679-1683. See also ref. [8b].

[12] This behaviour can be explained in terms of stereoelectronic effect: P.-Q. Huang, Synlett 2006, 1133-1149.

[13] For a review, see: a) T. S. Kaufman, Tetrahedron: Asymmetry 2004, 15, 1203-1237. For more recent work, see: b) T. Kanemitsu, Y. Yamashita, K. Nagata, T. Itoh, Synlett 2006, 1595-1597; c) F. Werner, N. Blank, T. Opatz, Eur. J. Org. Chem. 2007, 3911-3915; d) C. Li, J. Xiao, J. Am. Chem. Soc. 2008, 130, 13208-13209.

[14] For a review, see: A. B. J. Bracca, T. S. Kaufman, Tetrahedron 2004, $60,10575-10610$. 
[15] The $\alpha$-amidoalkylation failed when using $p$-methoxyphenylzinc iodide, lactam 2 being recovered along with variable amounts of isoquinolone $\mathbf{3}$.

[16] For previous enantioselective syntheses, see: a) A. Brossi, S. Teitel, Helv. Chim. Acta 1971, 54, 1564-1571; b) M. J. Munchhof, A. I. Meyers, J. Org. Chem. 1995, 60, 7086-7087; c) H. Suzuki, S. Aoyagi, C. Kibayashi, Tetrahedron Lett. 1995, 36, 6709-6712; see also refs. [5a,d].

[17] For previous enantioselective syntheses, see: a) G. Gosmann, D. Guillaume, H.-P. Husson, Tetrahedron Lett. 1996, 37, 4369-4372; b) V. Samano, J. A. Ray, J. B. Thompson, R. A. Mook, Jr., D. K. Jung, C. S. Koble, M. T. Martin, E. C. Bigham, C. S. Regitz, P. L. Feldman, E. E. Boros, Org. Lett. 1999, 1, 1993-1996; see also ref. [16a].

[18] For previous enantioselective syntheses, see: a) N. Yamazaki, H. Suzuki, S. Aoyagi, C. Kibayashi, Tetrahedron Lett. 1996, 37, 61616164; b) R. Pedrosa, C. Andrés, J. M. Iglesias, J. Org. Chem. 2001, 66, 243-250; c) P. Allef, H. Kunz, Heterocycles 2007, 74, 421-436; d) M. K. Pyo, D.-H. Lee, D.-H. Kim, J.-H. Lee, J.-C. Moon, K. C. Chang, H. S. Yun-Choi, Bioorg. Med. Chem. Lett. 2008, 18, 4110 4114.; see also ref. [13c].

[19] S. V. Kini, M. M. V. Ramana, Tetrahedron Lett. 2004, 45, 41714173

[20] For the enantioselective synthesis of the enantiomer, see: K. Th. Wanner, I. Praschak, Heterocycles 1989, 29, 29-33.

[21] In this series, the polyallylated compound 1-(2-allyl-2-hydroxy-4pentenyl)-2-[1-(2-hydroxy-1 $R$-phenylethylamino)-3-butenyl]-4,5dimethoxybenzene (10) was isolated as a by-product (39\%).

[22] Treatment of lactam 2 with allyltrimethylsilane in the presence of $\mathrm{TiCl}_{4}$ or with 2-(1,3-dioxolan-2-yl)ethylzinc iodide led to isoquinolone $\mathbf{3}$, the starting lactam being partly recovered in the latter case.

[23] For previous enantioselective syntheses, see: a) T. R. Wu, M. Chong, J. Am. Chem. Soc. 2006, 128, 9646-9647; b) J. Szawkato, S. J. Czarnocki, A. Zawadzka, K. Wojtasiewicz, A. Leniewski, J. K. Maurin, Z. Czarnocki, J. Drabowicz, Tetrahedron: Asymmetry 2007, 18, 406-413; c) S. M. Allin, S. N. Gaskell, J. M. R. Towler, P. C. B. Page, B. Saha, M. J. McKenzie, W. P. Martin, J. Org. Chem. 2007, 72, 8972-8975; d) T. Kanemitsu, Y. Yamashita, K. Nagata, T. Itoh, Heterocycles 2007, 74, 199-203; e) G.-H. Hou, J.-H. Xie, P.-C. Yan, Q.-L. Zhou, J. Am. Chem. Soc. 2009, 131, 1366-1367.

[24] a) T. Kametani in The Total Synthesis of Natural Products, Vol 3 (Ed.: J. ApSimon), Wiley, New York, 1977, pp.1-272; b) M. J. Munchhof, A. I. Meyers, J. Org. Chem. 1996, 61, 4607-4610. See also refs. [1a,1d].

[25] R. P. Polniaszek, C. R. Kaufman, J. Am. Chem. Soc. 1989, 111, 4859-4863.

[26] I. W. Southon, J. Buckingham, Dictionary of Alkaloids, Chapman and Hall, London, 1989, p 261. 


\section{Layout 2:}

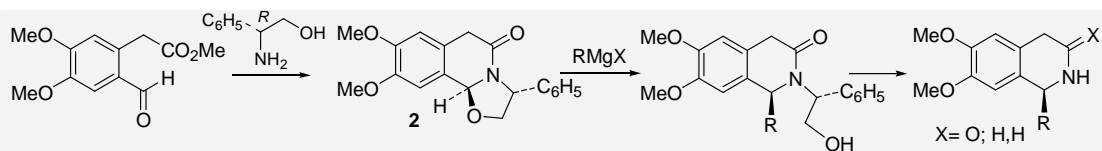

$\mathrm{R}=\mathrm{Me}$, Et, 3,4-(MeO) $)_{2} \mathrm{C}_{6} \mathrm{H}_{3}, 3,4,5-(\mathrm{MeO})_{3} \mathrm{C}_{6} \mathrm{H}_{2},(p-\mathrm{MeO}) \mathrm{C}_{6} \mathrm{H}_{4} \mathrm{CH}_{2}, \mathrm{C}_{6} \mathrm{H}_{5} \mathrm{CH}_{2} \mathrm{CH}_{2}, \mathrm{CH}_{2}=\mathrm{CHCH}_{2}$,

Tricyclic $\quad(R)$-phenylglycinol-derived lactam 2 has proven to be a versatile scaffold that provides general access to enantiopure 1-substituted tetrahydroisoquinoline derivatives as well as more complex alkaloids, e.g. (-)crispine $A$, bearing the tetrahydroisoquionoline moiety.
M. Amat, ${ }^{*}$ V. Elias, N. Llor, F. Subrizi, E. Molins, J. Bosch Page No. Page No.

A General Methodology for the Enantioselective Synthesis of 1Substituted Tetrahydroisoquinoline Alkaloids

Keywords: Alkaloids /

Tetrahydroisoquinolines / Lactams / Phenylglycinol / $\alpha$-Amidoalkylation 
